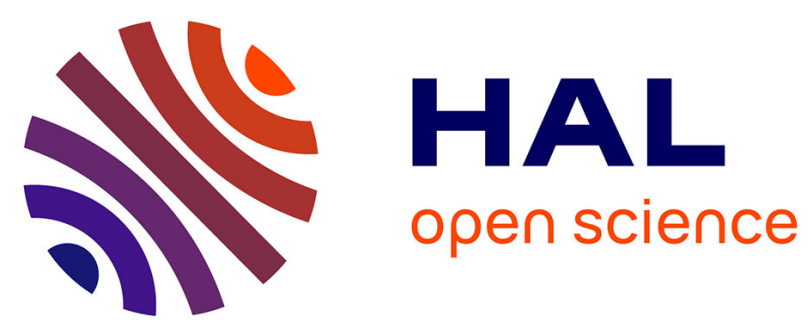

\title{
Transient E.S.R. absorption in Eu0.4Sr0.6S, in the paramagnetic phase, near the spin-glass temperature: the exchange reservoir
}

\author{
A. Deville, C. Blanchard, A. Landi
}

\section{- To cite this version:}

A. Deville, C. Blanchard, A. Landi. Transient E.S.R. absorption in Eu0.4Sr0.6S, in the paramagnetic phase, near the spin-glass temperature: the exchange reservoir. Journal de Physique, 1985, 46 (6), pp.965-978. 10.1051/jphys:01985004606096500 . jpa-00210044

\section{HAL Id: jpa-00210044 https://hal.science/jpa-00210044}

Submitted on 1 Jan 1985

HAL is a multi-disciplinary open access archive for the deposit and dissemination of scientific research documents, whether they are published or not. The documents may come from teaching and research institutions in France or abroad, or from public or private research centers.
L'archive ouverte pluridisciplinaire HAL, est destinée au dépôt et à la diffusion de documents scientifiques de niveau recherche, publiés ou non, émanant des établissements d'enseignement et de recherche français ou étrangers, des laboratoires publics ou privés. 
Classification

Physics Abstracts

$75.30-76.90$

\title{
Transient E.S.R. absorption in $\mathrm{Eu}_{0.4} \mathrm{Sr}_{0.6} \mathrm{~S}$, in the paramagnetic phase, near the spin-glass temperature : the exchange reservoir
}

\author{
A. Deville, C. Blanchard and A. Landi \\ Laboratoire d'Electronique des Milieux Condensés $\left({ }^{*}\right)$, Aix-Marseille I, 13397 Marseille Cedex 13, France
}

(Reçu le 23 juillet 1984, révisé le 20 décembre 1984, accepté le 7 février 1985)

\begin{abstract}
Résumé. - Nous avons étudié dans le verre de spin isolant $\mathrm{Eu}_{0,4} \mathrm{Sr}_{0,6} \mathrm{~S}$, en bande $\mathrm{X}$ et pour $T \gtrsim T$, le signal transitoire de résonance électronique consécutif à une impulsion intense de champ hyperfréquence. Ce signal dépend fortement des conditions expérimentales (énergie de l'impulsion hyperfréquence, valeur du champ magnétique directeur). A 4,2 K, pour une énergie perturbatrice faible $(1 \mu \mathrm{J})$, le retour à l'équilibre est exponentiel $(\tau \sim 40 \mathrm{~ms})$. En associant des expériences de saturation progressive de la raie de résonance à des expériences impulsionnelles effectuées à différentes valeurs du champ directeur, nous avons montré que le signal observé correspond à un déplacement transitoire de la raie d'absorption, associé à une élévation de la température de spin. Ce signal est finalement la manifestation dynamique d'un comportement déjà observé par résonance électronique en régime permanent.

Nous discutons les résultats expérimentaux à l'aide du modèle du réservoir d'échange, de Bloembergen et Wang. Nous trouvons que, grâce aux interactions dipolaires, l'énergie hyperfréquence absorbée par le réservoir Zeeman est rapidement $\left(10^{-10} \mathrm{~s}\right)$ transférée au réservoir d'échange. Cette énergie est ensuite cédée aux phonons grâce à la modulation du couplage d'échange par les phonons (processus direct). La capacité calorifique des spins provient essentiellement de l'énergie d'échange et, jusqu'à $15 \mathrm{~K}$, l'emporte sur celle des phonons, ce qui conduit à l'existence d'un goulot de phonons : à la fin de l'impulsion, les phonons prennent rapidement la température des spins, puis la température de l'ensemble du système spins-phonons relaxe lentement vers celle du bain.
\end{abstract}

\begin{abstract}
In the insulating spin-glass material $\mathrm{Eu}_{0.4} \mathrm{Sr}_{0.6} \mathrm{~S}$, the transient E.S.R. absorption following a strong r.f. pulse has been studied at $\mathrm{X}$ band for $T \gtrsim T_{\mathrm{g}}$. The signal was found to depend strongly upon the experimental conditions (energy of the r.f. pulse, value of the driving magnetic field). At $4.2 \mathrm{~K}$ and with a weak perturbing r.f. energy $(1 \mu \mathrm{J})$, the recovery was exponential $(\tau \sim 40 \mathrm{~ms})$. By coupling continuous saturation experiments with pulse experiments made at different values of the driving field, we have shown that the observed signal corresponds to a transient shift of the absorption line, reflecting a rise of the spin temperature. This signal is finally the dynamical manifestation of a behaviour already met in steady E.S.R. experiments.

The experimental results are discussed within the Bloembergen-Wang exchange reservoir model; it is found that, due to the dipolar interactions, the r.f. energy absorbed by the Zeeman reservoir is rapidly $\left(10^{-10} \mathrm{~s}\right)$ transferred to the exchange reservoir. The energy is then given up to the phonons through the modulation of the exchange coupling by the phonons (direct process). The heat capacity of the spins mainly comes from the exchange energy, and overwhelms that of the phonons up to $15 \mathrm{~K}$; this leads to a phonon-bottleneck : the phonons rapidly reach the spin temperature at the end of the pulse, then the temperature of the whole spin-phonon system slowly relaxes towards the bath temperature.
\end{abstract}

\section{Introduction.}

Since the discovery of a cusp in the a.c. susceptibility of AuFe alloys (Cannella and Mydosh, 1972 [1]) considerable experimental and theoretical efforts have been made in order to elucidate the nature of the spin-

(*) Unité Associée au C.N.R.S. : UA 784. glass state. E.S.R. can be detected in spin-glasses, and represents a powerful tool for their study, as it conveys a lot of information : the resonance field, the lineshape, linewidth and intensity of the absorption signal, and the way these parameters depend upon the temperature, and upon the frequency of the r.f. field.

Two important features of the E.S.R. in metallic spin-glasses - the shift of the resonance towards low fields, and the broadening of the absorption signal, 
when the temperature is lowered down to the freezing temperature $T_{\mathrm{g}}$ - were in fact observed in CuMn and described in pioneering papers $[2,3]$ by Owen $e t$ al. more than 25 years ago. Since that time, a similar behaviour has been observed in other metallic spinglasses (e.g. AgMn [4]) as well as in non-metallic spinglasses (e.g. Eu : $\mathrm{SrS}[5]$ ); in fact, insulating and metallic spin-glasses display somewhat different E.S.R. behaviour, but these differences are not related to the spinglass character, and originate from the presence of conduction electrons in metals : in a metallic spinglass, at first the absorption line is Dysonian at hightemperature, and secondly when the temperature is lowered one does not observe a monotonous increase of the linewidth, but rather the existence of a minimum width at some temperature $T_{\mathrm{m}}>T_{\mathrm{g}}$, which merely indicates that at high temperature the line is broadened by spin-lattice relaxation (Korringa process, leading to a width $\Delta H \propto T$ ).

Owen et al. attempted to explain their experimental observations by assuming the presence of a kind of antiferromagnetism. By now, it is clear that another explanation has to be found. Theories of the E.S.R. in spin-glasses have been recently developed [6-9]. We think that a better knowledge of the relaxation processes would be of interest for those trying to elucidate the nature of the E.S.R. in spin-glasses. With this idea in mind, we have undertaken a study of the dynamical aspects of E.S.R. in the insulating spin-glass $\mathrm{Eu}_{0.4} \mathrm{Sr}_{0.6} \mathrm{~S}$; in a previous paper [5], we have presented the static E.S.R. properties of this material in detail; in this paper, we present and discuss the dynamical results for $T \gtrsim T_{\mathrm{g}}\left(T_{\mathrm{g}}=1.55 \mathrm{~K}\right.$ at $\left.\omega=0\right)$. As far as we know, this is the first paper dealing with dynamical aspects of electron spin resonance in spin-glasses; on the contrary the dynamical aspects of the resonance of nuclei in spin-glasses have already been investigated (see for instance the zero-field N.M.R. study of iron-doped 2H-niobium diselenide, by Chen and Slichter [10a] and references therein, and [10b]). The reason for this difference is perharps that most experimental work concerns metallic spin-glasses, and that as already stated, spin-lattice relaxation associated with the presence of conduction electrons is effective in these materials; in $\mathrm{Eu}: \mathrm{SrS}$ on the contrary, of course this relaxation does not occur, and moreover the magnetic ion $\mathrm{Eu}^{2+}$ is only weakly coupled to the phonons by the spin-orbit coupling (S ground state). We present the system in section 2 ; the experimental conditions and results are given in section 3 ; it is shown that the recovery is quite different from that observed in conventional dilute paramagnetic materials. These results are discussed in section 4; we show why the Bloembergen-Wang exchange reservoir model should be used, and calculate the various parameters of the model (heat capacities and relaxation times); the numerical and the experimental results from both static and pulse E.S.R. lead to a simplified description of the system. In this paper most experimental results are obtained for $T>T_{\mathrm{g}}$, and we discuss only the case $T \gtrsim T_{\mathrm{g}}$.

\section{The system.}

EuS has the $\mathrm{NaCl}$ f.c.c. structure; each europium ion is surrounded by 6 sulfur ions, 12 n.n. and 6 n.n.n. europium ions, at $2.98,4.21$ and $5.96 \AA$ respectively; in $\mathrm{Eu}_{x} \mathrm{Sr}_{1-x} \mathrm{~S}$, it is thought that the europium ions substitute randomly for the strontium ions.

E.S.R. studies of europium diluted in diamagnetic SrS have established that in these materials, europium is in the divalent state and possesses a $7 / 2$ electron spin $\left(4 f^{7}\right.$ ground configuration, ${ }^{8} \mathrm{~S}$ ground state, $J=7 / 2$ ground multiplet). EuS orders ferromagnetically below $T_{\mathrm{c}}=16.7 \mathrm{~K}$. The exchange coupling is ferromagnetic between nearest neighbours, and antiferromagnetic between n.n. neighbours; writing $-2 J \mathbf{s}_{i} \mathbf{s}_{j}$ the exchange Hamiltonian between $\mathbf{s}_{i}$ and $\mathbf{s}_{j}$, the coupling constants are respectively $J_{1}$ and $J_{2}$ with $J_{1} / k=$ $+0.221 \pm 0.003 \mathrm{~K}$ and $J_{2} / k=-0.100 \pm 0.004 \mathrm{~K}$ [11]; our previous work on a spherical single crystal [5] showed that in $\mathrm{Eu}_{0.4} \mathrm{Sr}_{0.6} \mathrm{~S}$ the room temperature absorption spectrum consists of a single exchangenarrowed line (Lorentzian line, with $\Delta H_{\mathrm{pp}}=720 \mathrm{G}$, and $\left.g_{\text {eff }}=2.012 \pm 0.006\right)$ : one can disregard the hyperfine coupling, as it is far less important than the exchange and dipolar couplings, and is not resolved at this high europium concentration level; when the temperature was lowered, a broadening of the line and a shift of its centre towards low fields were observed below $30 \mathrm{~K}$; the broadening effect becomes less temperature dependent below $T=2.3 \mathrm{~K}$ which is close to the value $T_{\mathrm{g}}=2.2 \mathrm{~K}$ extrapolated from optical measurements and we proposed to identify this temperature with the freezing temperature at $9.3 \mathrm{GHz}$.

\section{Experimental conditions and results.}

Measurements were made on the spherical single crystals used in our previous E.S.R. work [5] $(\varnothing=$ $0.8 \mathrm{~mm})$, at $\mathrm{X}$ band $(9.3 \mathrm{GHz})$; the sample was on the bottom of a quartz tube, placed along the axis of a $\mathrm{TE}_{011}$ cylindrical copper cavity (diameter $46 \mathrm{~mm}$, unloaded quality factor $Q_{0} \sim 10^{4}$ ); the sample was in the middle of the cavity, and submitted to a r.f. magnetic field $2 H_{1} \cos \omega t$ directed along the cavity axis; at critical coupling, the amplitude of each circular component was $H_{1} \simeq 1.4 \sqrt{P}$ ( $H_{1}$ gauss, $P$ watt), $P$ being the r.f. power incident upon the cavity (Appendix 1). The cavity was placed in a bath cryostat and filled with liquid helium. The quartz tube was filled with liquid helium.

We used a superheterodyne single klystron spectrometer (0.5 W, V58 Varian klystron) [12], for both continuous and pulse saturation experiments. This spectrometer allows a direct detection of the absorption, which would be very difficult with a conventional homodyne spectrometer. In continuous saturation experiments, the absorption line was first recorded 
with a low incident power; successive recordings of the line were then made with increasing power upon the sample, each increase being compensated by an identical attenuation introduced between the cavity and the detection circuit : with this procedure, we directly recorded the absorption component $\chi^{\prime \prime}\left(H, H_{1}\right)$ of the susceptibility $(H:$ driving magnetic field) from now on, $\chi^{\prime \prime}$ will be called the susceptibility for brevity. In the pulse experiments, the driving field was kept constant, and the spins were submitted to a pulse of intense r.f. power (power $\boldsymbol{P}_{\text {sat }}$, duration $\left.\tau_{\text {sat }}\right)$; we then recorded the transient E.S.R. signal using a weak r.f. field (typically $1-10 \mu \mathrm{W}$ ) as a probe. We were able to add up several samples of the recovery signal (transient recorder DL 905 from DATALAB, followed by a multichannel analyser KVT 250 from C.R.C.).

With $\mathrm{Eu}_{0.4} \mathrm{Sr}_{0.6} \mathrm{~S}$, the transient E.S.R. signal observed at the end of the saturating pulse shows a dependence upon the various parameters (duration and power of the saturating pulse, value of the driving field) which is quite different from that in the case of a dilute paramagnet. We present the origin of this behaviour before going into the detail of the experimental results :

- in a conventional paramagnet, considering for simplicity the simplest case (crystalline solid, with neither fine nor hyperfine structure, homogeneous line), the susceptibility at two temperatures $T^{\prime}$ and $T^{\prime \prime}>T^{\prime}$ has the shape given in figure 1a (Curie law); in a pulse experiment, starting from a spin temperature $T_{\mathrm{S}}=T^{\prime}$, it is possible to raise the spin temperature if the duration and power of the pulse are well chosen. The transient signal reflects the thermal variation of the susceptibility, and is identical for symmetrical values of the driving field around the resonance field (Fig. 1 $\left.a^{\prime}\right)$;

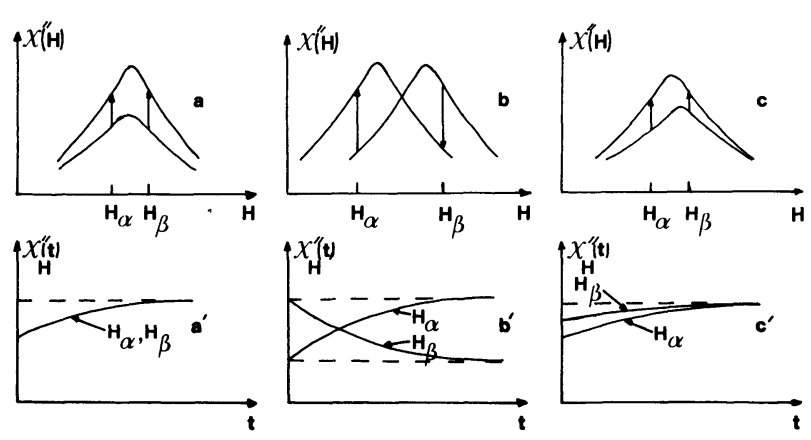

Fig. 1. - Method for the discrimination between a) temporary decrease of absorption b) and c) temporary shift of the E.S.R. line ; a) is obtained with a usual dilute paramagnet, b) with $\mathrm{Eu}_{0.4} \mathrm{Sr}_{0.6} \mathrm{~S}$ around $1.6 \mathrm{~K}$ and c) with $\mathrm{Eu}_{0.4} \mathrm{Sr}_{0.6} \mathrm{~S}$ around $4.2 \mathrm{~K}$.

- in $\mathrm{Eu}_{0.4} \mathrm{Sr}_{0.6} \mathrm{~S}$, the position and width of the E.S.R. line is strongly temperature dependent [5] : below $30 \mathrm{~K}$, when the temperature is lowered, the line is shifted towards lower fields, and broadens; this has consequences in pulse experiments : the shapes of the absorption lines at $1.6 \mathrm{~K}$ and at a slightly higher temperature are given in figure $1 \mathrm{~b}$; in corresponding pulse experiments, the transient E.S.R. signal recorded (Fig. $1 b^{\prime}$ ) for the values $H_{\alpha}$ and $H_{\beta}$ of the driving field (Fig. 1b) will have opposite time variations. The shapes of the absorption line at $4.2 \mathrm{~K}$ and at a slightly higher temperature, and that of the transient signal around the resonance field are given in figures $1 \mathrm{c}$ and $1 \mathrm{c}^{\prime}$ respectively. It was the observation of this quite unusual behaviour, most spectacular around $1.6 \mathrm{~K}$, which led us to realize that the temperature dependence of the E.S.R. line was important in pulse as well as in static experiments. In static experiments, the spin system and the bath are at the same temperature, but the magnetization and the resonance frequency finally depend upon the temperature of the spin system (spin temperature); in the pulse experiments, the photons from the r.f. field raise the spin temperature, and a temporary decrease of the shift of the resonance line must result. We were finally able to give a direct proof of this behaviour by adding, to the usual pulse measurements at the resonance field, continuous saturation measurements coupled to pulse experiments made at various driving fields. We now present our experimental results in more detail.

Most experimental results presented hereafter have been established at $4.2 \mathrm{~K}$, and some around $1.6 \mathrm{~K}$. Some experiments were somewhat delicate (continuous saturation experiments, pulse experiments with $P_{\text {sat }} \cdot \tau_{\text {sat }} \leqslant 10^{-6} \mathrm{~J}$ ) so we did not try, for instance, to determine at which temperature one changes from the behaviour of figure $1 \mathrm{~b}$, to that of figure $1 \mathrm{c}$. We first present the main results from continuous saturation experiments at $4.2 \mathrm{~K}$ : the susceptibility $\chi^{\prime \prime}\left(H, H_{1}\right)$ and its derivative, for the incident power $P=10 \mu \mathrm{W}$, and for $P=100 \mathrm{~mW}$, are given in figure 2 ; from the

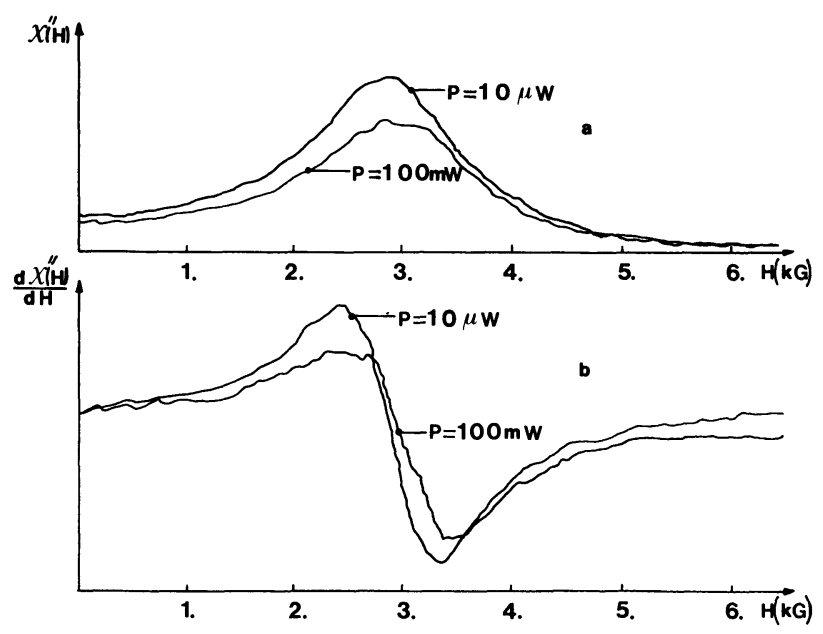

Fig. 2. - a) Absorption component $\chi^{\prime \prime}(H)$ of the susceptibility for $\mathrm{Eu}^{2+}$ in $\mathrm{Eu}_{0.4} \mathrm{Sr}_{0.6} \mathrm{~S}$ at $4.2 \mathrm{~K}$, for $P=10 \mu \mathrm{W}$ and $P=100 \mathrm{~mW}$, using the same arbitrary units for both recordings. b) Derivative $d \chi^{\prime \prime}(H) / d H$ for the same sample and conditions, using the same arbitrary units for both recordings (modulation field : $60 \mathrm{G}_{\mathrm{pp}}, 29 \mathrm{~Hz}$ ). 
variation of the shift of the E.S.R. line and our previous results [5], we find that the spin temperature increase for $P=100 \mathrm{~mW}$ is roughly $\Delta T_{\mathrm{s}} \simeq 0.9 \mathrm{~K}$; this will be the maximum temperature increase at the end of a $100 \mathrm{~mW}$ saturating pulse.

We now present the results obtained from pulse experiments at $4.2 \mathrm{~K}$ : we first show the shape of the recovery signal at various driving fields, then study its dependence upon the duration and power of the r.f. pulse, at the resonance field. The transient E.S.R. signal obtained at $4.2 \mathrm{~K}$ at the end of a saturating pulse $\left(P_{\text {sat }}=100 \mathrm{~mW}, \tau_{\text {sat }}=1 \mathrm{~ms}\right)$ is given in figure 3 for

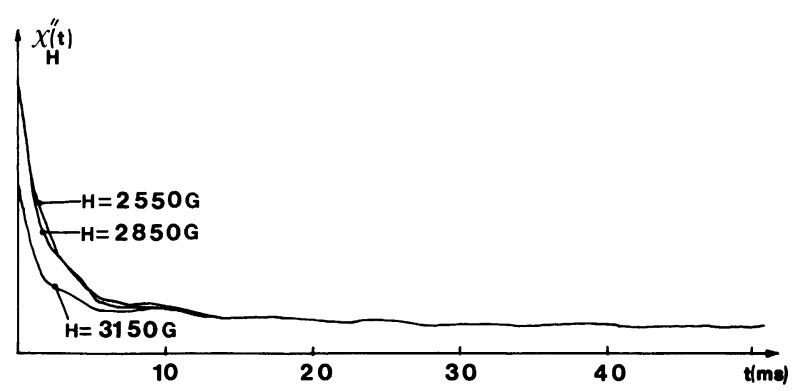

Fig. 3. - Transient E.S.R. absorption signal at $4.2 \mathrm{~K}$, for $\mathrm{Eu}^{2+}$ in $\mathrm{Eu}_{0.4} \mathrm{Sr}_{0.6} \mathrm{~S}$ after a pulse with $P_{\text {sat }}=100 \mathrm{~mW}$, $\tau_{\text {sat }}=1 \mathrm{~ms}$, for different values of the driving field : $2850 \mathrm{G}$ (resonance field), 2550 and $3150 \mathrm{G}$.

different values of the driving field : the signal is nearly the same for the resonance field $(2850 \mathrm{G})$ and for $H=2550 \mathrm{G}$, while it has the same shape but a lower intensity when $H=3150 \mathrm{G}$; all this is consistent with the continuous saturation results of figure $2 \mathrm{a}$. All the following results at $4.2 \mathrm{~K}$ were obtained at $H=2850 \mathrm{G}$; we observed that the recovery signal depends only upon the energy $P_{\text {sat }} \cdot \tau_{\text {sat }}$ of the r.f. pulse (in our experiments $\tau \lesssim 100 \mathrm{~ms}$ ); this is shown in figure $4 \mathrm{a}\left(10^{-5} \mathrm{~J}\right)$ and $4 \mathrm{~b}\left(10^{-6} \mathrm{~J}\right)$. We stress the

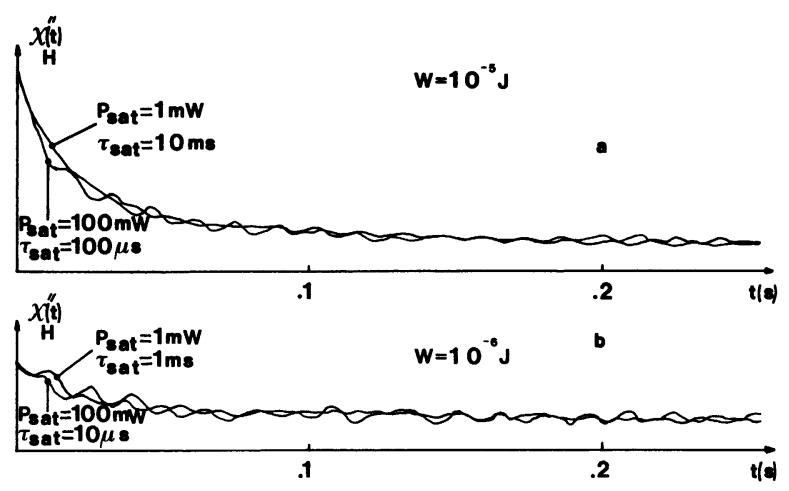

Fig. 4. - Transient E.S.R. absorption signal at $4.2 \mathrm{~K}$ and $H=2850 \mathrm{G}$, for $\mathrm{Eu}^{2+}$ in $\mathrm{Eu}_{0.4} \mathrm{Sr}_{0.6} \mathrm{~S}$ after a) a $10^{-5} \mathrm{~J}$ pulse b) a $10^{-6} \mathrm{~J}$ pulse. In fact, a) is a summation of 60 and b) of 200 scans; both recordings use the same arbitrary units, showing that the intensities at the end of a single pulse are roughly in a ratio of 10 . fact that even a pulse as short as $10 \mu \mathrm{s}$ is able to disturb the spin system, if $P_{\text {sat }}$ is high enough. The recovery signal for $P_{\text {sat }}=1 \mathrm{~mW}$ and $\tau_{\text {sat }}=1,10$ and $100 \mathrm{~ms}$ is presented in figure $5:$ its intensity at

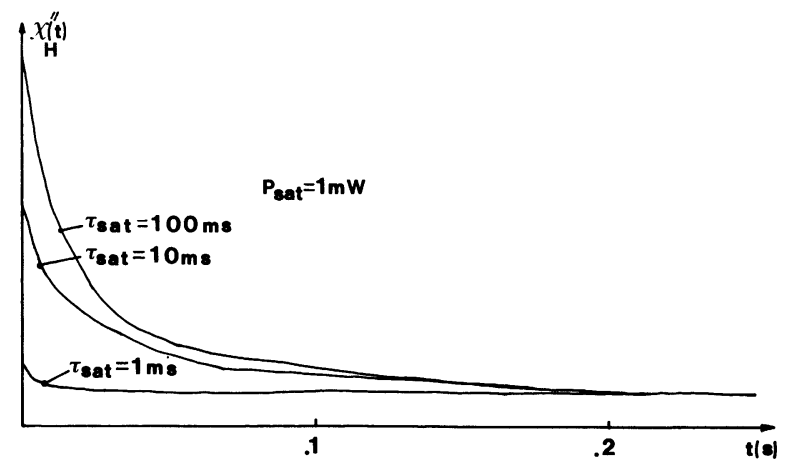

Fig. 5. - Transient E.S.R. absorption signal at $4.2 \mathrm{~K}$ and $2850 \mathrm{G}$, for $\mathrm{Eu}^{2+}$ in $\mathrm{Eu}_{0.4} \mathrm{Sr}_{0.6} \mathrm{~S}$ after a pulse with $P_{\text {sat }}=$ $1 \mathrm{~mW}$ and $\tau_{\text {sat }}=1,10$ and $100 \mathrm{~ms}$ (65 scans, same arbitrary units).

the end of the pulse increases roughly linearly from 1 to $10 \mathrm{~ms}$, while the intensity ratio for the signals obtained with 100 and $10 \mathrm{~ms}$ is less than 2 (this is probably related to the fact that the relaxation time constant of the spin system is $40 \mathrm{~ms}$ ). These recovery signals are shown in more detail in figures 6,7 and $8:$ the transient absorption for $\tau_{\text {sat }}=100 \mathrm{~ms}$ is presented in figure $6 \mathrm{a}:$ it has a non-exponential behaviour and can be fitted with the analytical expression $y(t)=$ $0.3 \mathrm{e}^{-t / 5}+0.7 \mathrm{e}^{-t / 40}(t: \mathrm{ms})$; figure $6 \mathrm{~b}$, obtained
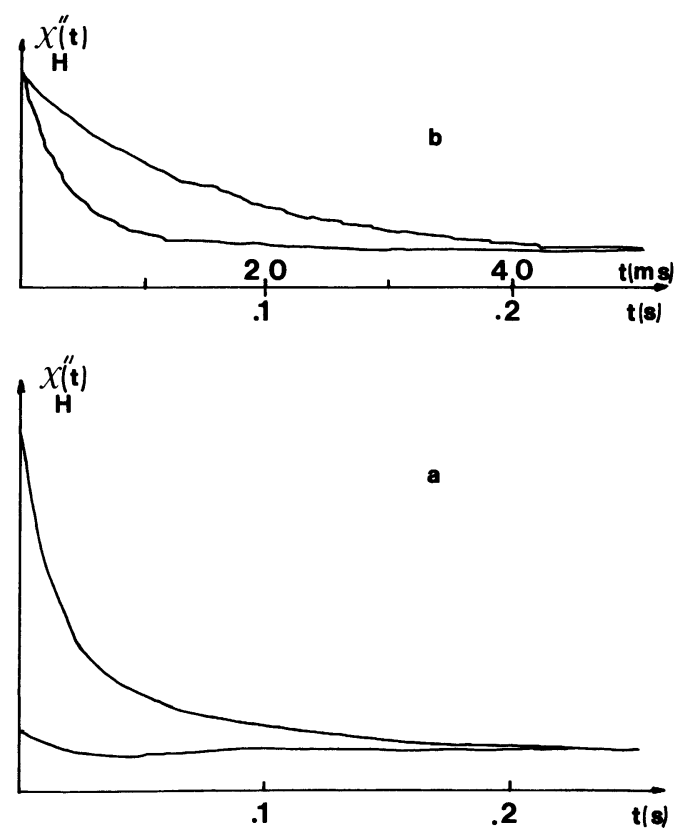

Fig. 6. - Transient E.S.R. absorption signal at $4.2 \mathrm{~K}$ and $H=2850 \mathrm{G}$, for $\mathrm{Eu}^{2+}$ in $\mathrm{Eu}_{0.4} \mathrm{Sr}_{0.6} \mathrm{~S}$ after a pulse with $P_{\text {sat }}=1 \mathrm{~mW}, \tau_{\text {sat }}=100 \mathrm{~ms}:$ a) we subtracted from the upper curve $\left(\mathrm{Eu}^{2+}\right)$ the lower curve (spurious signal) b) the $\mathrm{Eu}^{2+}$ signal was recorded with two different time bases : $51.2 \mathrm{~ms}$ scan (upper curve) and $256 \mathrm{~ms}$ scan (lower curve). 


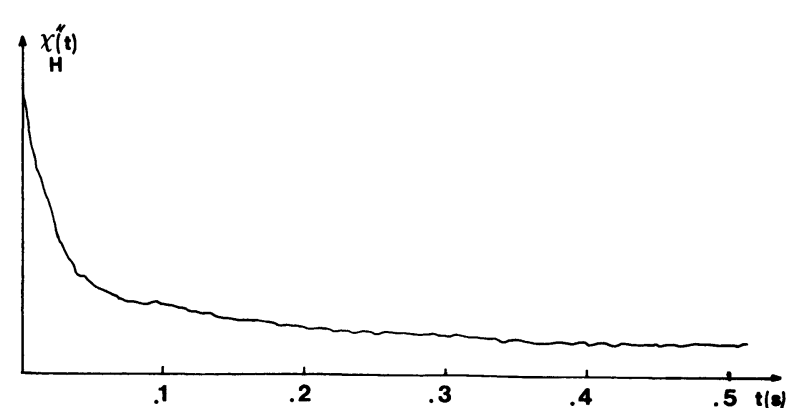

Fig. 7. - Same sample and conditions as in figure 6, except that $\tau=10 \mathrm{~ms}$.

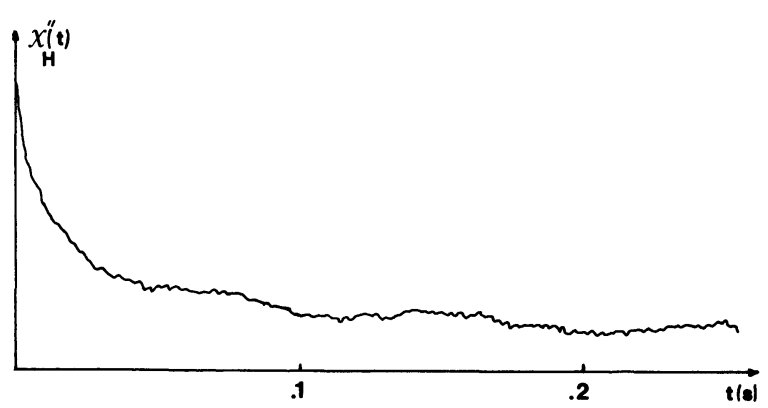

Fig. 8. - Same sample and conditions as in figure 6, except that $\tau=1 \mathrm{~ms}$.

with a better time resolution, clearly shows the existence of the shorter time constant $(5 \mathrm{~ms})$. The transient signal for $\tau_{\text {sat }}=10 \mathrm{~ms}$ is given in figure 7 , and fits with an expression $y(t)=0.2 \mathrm{e}^{-t / 5}+0.8 \mathrm{e}^{-t / 40}$. The corresponding signal for $\tau=1 \mathrm{~ms}$ is given in figure 8 : within experimental error, it fits with an expression $y(t)=\mathrm{e}^{-t / 40}$. We will retain the result that when the spin system is only weakly perturbed, the relaxation is characterized by a single time constant, $40 \mathrm{~ms}$, and will keep it as characteristic of the relaxation of the system at $4.2 \mathrm{~K}$. We were not able to give an interpretation for the shorter time constant observed for a stronger perturbation of the spins.

We now present the results at $T \sim 1.6 \mathrm{~K}$. The susceptibility $\chi^{\prime \prime}\left(H, H_{1}\right)$ and its derivative, for the incident power $P=10 \mu \mathrm{W}$ and for $P=100 \mathrm{~mW}$ are given in figure 9; the spin temperature increase is now $\Delta T_{\mathrm{s}} \simeq 2.2 \mathrm{~K}$. The corresponding transient E.S.R. signals obtained at that temperature for two values $H_{\alpha} \sim 1300 \mathrm{G}$ and $H_{\beta} \sim 3300 \mathrm{G}$ far from the resonance field $\left(H_{0} \sim 2300 \mathrm{G}\right)$, and given in figure 10, were obtained using a magnetron $\left(P_{\text {sat }}=100 \mathrm{~W}, \tau_{\text {sat }}=1 \mu \mathrm{s}\right):$ this shows that it is possible to disturb the spin system even with a $1 \mu \mathrm{s}$ pulse (in the same magnetron experiments at $4.2 \mathrm{~K}$, the signal had a poor $\mathbf{S} / \mathrm{N}$ ratio and is not shown). We did not study this recovery signal in more detail; we however observed that the characteristic time is about $3 \mathrm{~ms}$ (see for instance the time scale in Fig. 10). Systematic measurements at various temperatures

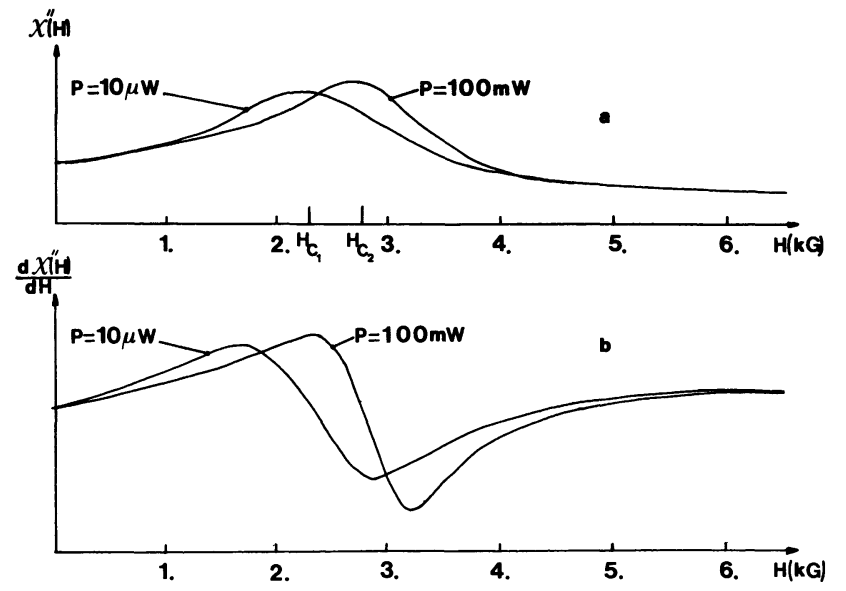

Fig. 9. - a) Absorption component $\chi^{\prime \prime}(H)$ of the susceptibility for $\mathrm{Eu}^{2+}$ in $\mathrm{Eu}_{0.4} \mathrm{Sr}_{0.6} \mathrm{~S}$ at $1.6 \mathrm{~K}$, for $P=10 \mu \mathrm{W}$ and $P=100 \mathrm{~mW}$, using the same arbitrary units for both recordings. b) Derivative $\mathrm{d} \chi^{\prime \prime}(H) / \mathrm{d} H$ for the same sample and conditions, using the same arbitrary units for both recordings (modulation field : $60 \mathrm{G}_{\mathrm{pp}}, 29 \mathrm{~Hz}$ ).

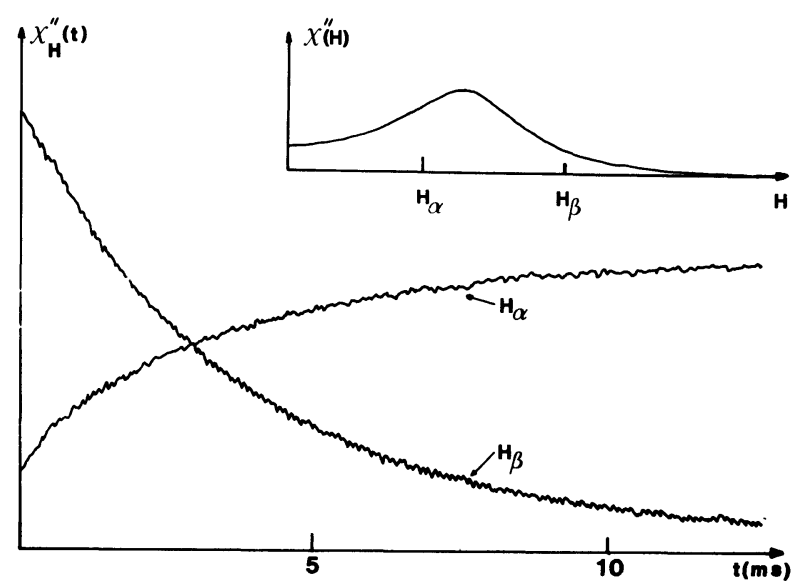

Fig. 10. - Transient E.S.R. absorption signal at $1.6 \mathrm{~K}$, for $\mathrm{Eu}^{2+}$ in $\mathrm{Eu}_{0.4} \mathrm{Sr}_{0.6} \mathrm{~S}$, after a pulse with $P_{\text {sat }}=100 \mathrm{~W}$, $\tau_{\text {sat }}=1 \mu \mathrm{s}$ (magnetron); $H_{\alpha} \simeq 1300 \mathrm{G}, H_{\beta} \simeq 3300 \mathrm{G}$; the same results where obtained with $P_{\text {sat }}=0.1 \mathrm{~W}, \tau_{\text {sat }}=$ $1 \mathrm{~ms}$ (klystron). If $H_{\mathrm{c} 1}<H<H_{\mathrm{c} 2}$, the transient signal is found to have a non monotonic time variation, as expected.

between 4.2 and $1.6 \mathrm{~K}$ need still be made to see how the observations at $1.6 \mathrm{~K}$ are correlated with those at $4.2 \mathrm{~K}$.

Before discussing the previous results, we point out that ordinary sample heating of the sample must be ruled out, for several reasons : dielectric losses are negligeable (insulator), the transient shift occurs within $1 \mu \mathrm{s}$, the thermal capacity of the spins is greater than that of the phonons $(\S 4)$.

Up to now, we have excluded the more complex behaviour which is observed when a higher energy is used : figure 11 shows for instance that at $2.1 \mathrm{~K}$ the recovery after a $10^{-2} \mathrm{~J}$ pulse proceeds through 


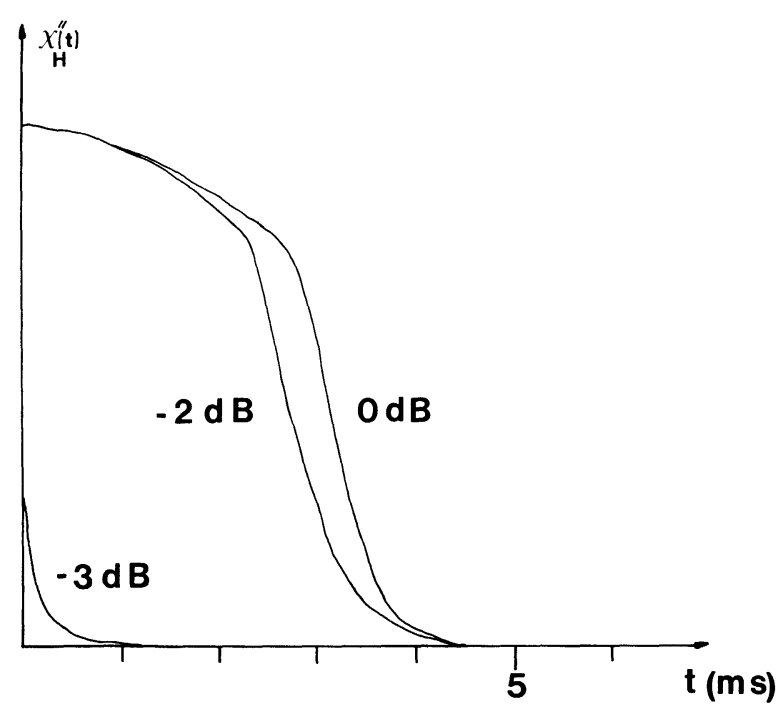

Fig. 11. - Pathological behaviour of the E.S.R. relaxation of $\mathrm{Eu}^{2+}$ in $\mathrm{Eu}_{0.4} \mathrm{Sr}_{0.6} \mathrm{~S}$ after a pulse with a high energy $\left(P_{\text {sat }}=100 \mathrm{~mW}\right.$ at $\left.0 \mathrm{~dB}, \tau_{\text {sat }}=100 \mathrm{~ms}\right) ; T=2.1 \mathrm{~K}$, $H=2500 \mathrm{G}$.

two steps. At $4.2 \mathrm{~K}$, in static experiments, a $0.2 \mathrm{~W}$ r.f. power is enough for creating a trough in the centre of the absorption curve, corresponding to a sudden spin temperature variation; we will not discuss these situations.

In the following discussion, we will first focus our interest on the interpretation of the time constant $(40 \mathrm{~ms})$ measured at $4.2 \mathrm{~K}$ for a weak perturbation of the spin system. This will lead us to introduce the exchange reservoir model, and to consider a phonon bottleneck; we will then discuss whether the observed spin temperature increase agrees with the predictions of the model.

\section{Discussion.}

We now discuss the physical origin of the transient E.S.R. signal. We first show that the exchange coupling is more efficient than the spin-phonon coupling, and that this signal cannot originate from a saturation of the exchange-narrowed line. This leads us to describe our system with the Bloembergen-Wang exchange reservoir model : we estimate the various parameters of the model.

4.1 ReLATIVE EFFICIENCIES OF THE SPIN-PHONON AND EXCHANGE COUPLINGS. - It is difficult to make predictions about the transient signal that one should get at $4.2 \mathrm{~K}$, which is near $T_{\mathrm{g}}$, for the corresponding theory is still lacking. It is however possible to get information by considering two simpler situations : $\mathrm{SrS}$ doped with a low concentration of $\mathrm{Eu}^{2+}$ ions, and $\mathrm{Eu}_{0.4} \mathrm{Sr}_{0.6} \mathrm{~S}$ in the paramagnetic regime far above $T_{\mathrm{g}}(T \gtrsim 30 \mathrm{~K})$. In the high dilution case (for instance a $10^{-4} \mathrm{Eu}^{2+}$ concentration), the transient signal is associated with the transfer of energy from each spin to the phonons, which act as a thermal bath (temperature $T$ ). It is reasonable to think that the time constant $T_{1}$ has neighbouring values in $\mathrm{SrS}: \mathrm{Eu}$ and in $\mathrm{CaF}_{2}$ : Eu, a material studied by Huang [13], who found $T_{1}^{-1}=12 T+5.5 \times 10^{-4} T^{5}\left(T_{1} \mathrm{~s}, T \mathrm{~K}\right)$; one then gets a direct process $\left(T_{1}^{-1} \propto T\right)$ if $T<10 \mathrm{~K}$, and a Raman process within the ground multiplet $\left(T_{1}^{-1} \propto T^{5}\right)$ if $T>10 \mathrm{~K}$. Huang found that $T_{1}=20 \mathrm{~ms}$ at $4.2 \mathrm{~K}$ and $75 \mu$ s at $30 \mathrm{~K}$. As we will need the $T_{1}$ value at $300 \mathrm{~K}$, we now extrapolate it from Huang's results : the right theoretical expression for this Raman process, valid over the whole temperature range and agreeing with Huang's experimental results obtained between 1.3 and $30 \mathrm{~K}$, is :

$$
T_{1}^{-1}=5.3 \times 10^{-4} T^{5} \frac{I_{4}\left(T_{\mathrm{D}} / T\right)}{I_{4}(+\infty)}\left(T_{1} \mathrm{~s}, T \mathrm{~K}\right)
$$

where

$$
I_{4}\left(x_{0}\right)=\int_{0}^{x_{0}} \frac{x^{4} \mathrm{e}^{x} \mathrm{~d} x}{\left(\mathrm{e}^{x}-1\right)^{2}}
$$

and $T_{\mathrm{D}}$ is the Debye temperature; a reasonable value for $T_{\mathrm{D}}$ is $T_{\mathrm{D}}=230 \mathrm{~K}\left({ }^{1}\right)[14,15]$; as $I_{4}(+\infty) \simeq 26$ and $I_{4}(0.8)=0.16$, we find that $T_{1}=1.3 \times 10^{-7} \mathrm{~s}$ at $300 \mathrm{~K}$.

We now consider $\mathrm{Eu}_{0.4} \mathrm{Sr}_{0.6} \mathrm{~S}$ in the temperature range $T \gtrsim 30 \mathrm{~K}$, where the broadening and shift of the E.S.R. line are negligible - the usual paramagnetic regime. The point to be examined is whether we are able to saturate the resonance at the lowest temperature $T=30 \mathrm{~K}$. One has to know the way the spin system returns to equilibrium after a perturbation, and the response of the system to a r.f. field of high amplitude. As the dipolar linewidth of $\mathrm{Eu}_{0.4} \mathrm{Sr}_{0.6} \mathrm{~S}$ is exchange-narrowed in the paramagnetic regime, the use of the Bloch-WangsnessRedfield theory (B.W.R.) $[16,17]$, which successfully describes the motional narrowing in liquids, is appropriate. We will limit ourselves to the case of a fluctuating dipolar field, as it corresponds to the present situation : the B.W.R. theory considers that the dipolar field created on a given spin by neighbouring spins fluctuates in time because of motion (or exchange), and the degrees of freedom associated with this motion are supposed to be in thermal equilibrium (bath). We recall the main results obtained with the two following assumptions :

1) if a given spin interacts with more than one spin, the motions of these neighbouring spins are uncorrelated;

2) all the correlation functions have the same correlation time $\tau_{\mathrm{c}^{\cdot}}$. Calling $\left\langle\Delta \omega^{2}\right\rangle$ the second

( $\left.{ }^{1}\right)$ Moruzzi et al. have found $T_{\mathrm{D}}=208 \mathrm{~K}$ for EuS, from specific heat measurements [15]; in [14], C. Arzoumanian et al. have proposed $T_{\mathrm{D}}=226 \mathrm{~K}$ for $\mathrm{Eu}_{0.54} \mathrm{Sr}_{0.56} \mathrm{~S}$ from unpublished sound velocities data. 
moment of the rigid lattice resonance line (dipolar linewidth), then if $\left\langle\Delta \omega^{2}\right\rangle^{1 / 2} \tau_{\mathrm{c}} \ll 1$ (narrowing condition) it is found that the longitudinal and transverse components of the magnetization relax in an exponential way, with time constants $T_{1}$ and $T_{2}$ respectively; the Bloch equations are then valid and the absorption line has the Lorentz shape; moreover when $\tau_{\mathbf{c}}$ is short enough to also satisfy $\omega_{\mathbf{z}} \tau_{\mathbf{c}} \ll 1$ (extreme narrowing), where $\omega_{\mathbf{Z}}$ is the Zeeman angular frequency, then $T_{1}=T_{2}$. As a consequence $T_{1}$ has a minimum value when $\omega_{\mathrm{z}} \tau_{\mathrm{c}}=1$. In the B.W.R. theory, it is moreover assumed that $\tau_{\mathrm{c}} \ll T_{1}, T_{2}$.

The linewidth behaves as follows : if $\left\langle\Delta \omega^{2}\right\rangle^{1 / 2} \tau_{\mathrm{c}} \lesssim$ $1<\omega_{\mathrm{z}} \tau_{\mathrm{c}}$ (moderate narrowing) the linewidth originates from the secular part $\mathscr{H}_{\mathrm{ds}}$ of the dipolar Hamiltonian $\mathfrak{H}_{\mathrm{d}}$, i.e. that part of $\mathfrak{H}_{\mathrm{d}}$ which commutes with the Zeeman Hamiltonian (adiabatic approximation, which is also used in the theoretical determination of $\left\langle\Delta \omega^{2}\right\rangle$ ) and the width is $\left\langle\Delta \omega^{2}\right\rangle \tau_{\mathrm{c}}$ (exchange narrowing). If $\omega_{\mathrm{z}} \tau_{\mathrm{c}} \lesssim 1$ (extreme narrowing), the non-secular part $\mathcal{H}_{\mathrm{dns}}$ also contributes to the linewidth (failure of adiabatic approximation) and the width is $\frac{10}{3}\left\langle\Delta \omega^{2}\right\rangle \tau_{c}[17]$

The behaviour of $T_{1}$ and $T_{2}$ associated with the fluctuations of the dipolar field are summarized in figure 12. In the presence of a narrowing, $T_{2}$ can be

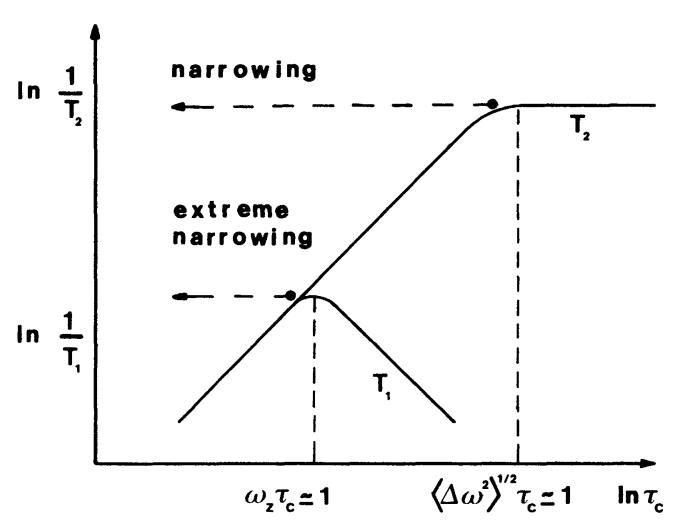

Fig. 12. - Qualitative behaviour of $T_{1}$ and $T_{2}$, the relaxation time constants of the longitudinal and transverse components of magnetization, versus $\tau_{c}$, the correlation time of the fluctuations of the dipolar field, in the BlochWangsness-Redfield theory of motional narrowing; $\omega_{\mathbf{z}}$ is the Zeeman angular frequency, $\left\langle\Delta \omega^{2}\right\rangle$ is the second moment of the rigid lattice resonance line.

obtained from the experimental absorption line : $T_{2}=\frac{2}{\gamma \Delta H}=\frac{2}{\sqrt{3} \gamma \Delta H_{\mathrm{pp}}}$, where $\Delta H$ and $\Delta H_{\mathrm{pp}}$ are the full width at half-height of the absorption line, and the width between the peak values of its derivative, respectively ; in the paramagnetic regime of $\mathrm{Eu}_{0.4} \mathrm{Sr}_{0.6} \mathrm{~S}$, we are roughly in the case of extreme narrowing $\left(\tau_{\mathrm{c}}=\frac{1}{\omega_{\mathrm{e}}}=6 \times 10^{-12} \mathrm{~s}[5] ; \omega_{\mathrm{e}}\right.$ is called the exchange angular frequency); then $T_{1}=T_{2}=9 \times 10^{-11} \mathrm{~s}$ $\left(\Delta H_{\mathrm{pp}}=720 \mathrm{G}\right)$. Comparing the present value $T_{1} \sim 10^{-10} \mathrm{~s}$ with the previous room-temperature value $T_{1}=1.3 \times 10^{-7} \mathrm{~s}$ for the relaxation time of single ions via the modulation of the crystal field by the phonons, we see that, in the temperature range $30 \mathrm{~K}-300 \mathrm{~K}$, the relaxation via the phonons should be far less efficient than the relaxation from the modulation of the dipolar field by exchange. It is therefore justified to examine the behaviour of the exchange narrowed line in the presence of a strong r.f. field. We will assume that the Bloch equations are still valid in the presence of such a strong field; this has been observed to be true in the E.S.R. of other exchange-narrowed lines in solids [18]. Then, half saturation of the absorption line $\left(\gamma^{2} H_{1}^{2} T_{1} T_{2}=1\right)$ would require a r.f. field $H_{1} \simeq 600 \mathrm{G}$ which is well beyond the maximum value of the field one can get from the klystron $\left(H_{1} \sim 1.0 \mathrm{G}\right.$ for $\left.P=0.5 \mathrm{~W}\right)$.

The previous discussion clearly demonstrates that the transient signal at $4.2 \mathrm{~K}$ is certainly not associated

- with the relaxation of the magnetization via the spin-phonon coupling, as this coupling is bypassed by the exchange coupling,

- with the relaxation of the magnetization via the modulation of the dipolar field by exchange, as the corresponding time is far shorter $\left(10^{-10} \mathrm{~s}\right)$ than the experimental time $(40 \mathrm{~ms})$.

Since exchange plays a major role in the relaxation of our system, we will use the Bloembergen-Wang exchange reservoir model. Incidently, we will have to understand how it is possible to temporarily raise the spin temperature, as the half-saturation of the exchange narrowed line seems to need a very high r.f. field.

Before turning to this discussion, let us finally note that the Waller mechanism (modulation of the dipolar interactions by the phonons) must also be ruled out, although we deal with a concentrated material : using the expression given in [19] for this mechanism and a direct process, with $\rho=5.8 \mathrm{~g} / \mathrm{cm}^{3}, n=7.6 \times$ $10^{21} \mathrm{spins} / \mathrm{cm}^{3}, v=2.9 \times 10^{5} \mathrm{~cm} / \mathrm{s}[14], \mu=\sqrt{63}$ Bohr magnetons, one finds that, at $4.2 \mathrm{~K}, T_{1} \sim 40 \mathrm{~s}$ for the direct process, which overcomes the Raman process up to $20 \mathrm{~K}$.

4.2 THE EXCHANGE RESERVOIR MODEL. - In the previous section, we have shown that the modulation of the crystal field, or that of the dipolar interactions, by the phonons, cannot explain our experimental observations. In fact, one must take the exchange coupling into account. Exchange alone would however be ineffective, and has to be considered together with the dipolar coupling. In this section we show that the use of the exchange reservoir model seems appropriate. This model was introduced by Bloembergen and Wang [20], and its quantum justification 
was given by Van Vleck [21]. Although it was then $\left(^{2}\right)$ used for ferromagnets in the paramagnetic region, we think that it can constitute a starting point for the spin glass $\mathrm{Eu}_{0.4} \mathrm{Sr}_{0.6} \mathrm{~S}$ when $T \gtrsim T_{\mathrm{g}}$, since it is based upon commutation relations which are still verified in a spin-glass. We first present and discuss the model, then make numerical estimates of several important parameters of this model.

4.2.1 Comments on the use of reservoir models. Let us first consider the Hamiltonian $\mathscr{H}=H_{\mathrm{z}}+\mathscr{H}_{\mathrm{Ex}}$, where $\mathscr{H}_{\mathrm{z}}$ and $\mathscr{H}_{\mathrm{Ex}}$ are the Zeeman and exchange Hamiltonians, respectively; the $\mathbf{g}$ tensor and $\mathfrak{H}_{\mathbf{E x}}$ are supposed to be isotropic, which is the case for $\mathrm{Eu}: \mathrm{SrS}$ (cubic symmetry); $\mathscr{H}_{\mathrm{z}}=g \beta_{\mathrm{e}} H S_{\mathrm{z}}$ with $S_{z}=\sum_{i=1}^{N_{0}} s_{z i}, \mathscr{H}_{\mathrm{Ex}}=-\sum_{i \neq j, j} J_{i j} s_{i} s_{j}$. Since $\left[\mathscr{H}_{\mathrm{Z}}, \mathscr{H}_{\mathrm{Ex}}\right]=0$, it is judicious to consider the eigenstates common to $\mathbf{S}^{2}=\left(\sum \mathbf{s}_{i}\right)^{2}$ and to $S_{z}=\sum s_{z i}$, which write $\left|S, M_{\mathrm{s}}\right\rangle$, where $S=N_{0} s, \quad N_{0} s-1, \ldots,-N_{0} s$; the spin-r.f. field Hamiltonian writes $\mathscr{H}_{\text {r.f. }}=$ $g \beta_{\mathrm{e}} 2 H_{1} \sum s_{x_{i}} \cos \omega t$. As $\left[S_{x}, S_{z}\right] \neq 0, \mathscr{H}_{\mathrm{r} . \mathrm{f} .}$ induces transitions between levels with different values of $M_{S}\left(\Delta M_{S}= \pm 1\right)$; on the contrary $\left[S_{x}, s_{i} s_{j}\right]=0$. Since in $\mathrm{Eu}_{0.4} \mathrm{Sr}_{0.6} \mathrm{~S}$ the exchange energy is important compared to the Zeeman and dipolar energies, and since $\left[\mathcal{H}_{\mathrm{z}}, \mathcal{H}_{\mathrm{Ex}_{\mathbf{x}}}\right]=0$, it is possible to consider two distinct reservoirs, the Zeeman reservoir, associated with $\mathscr{H}_{\mathrm{z}}$, and the exchange reservoir, associated with $\mathcal{H}_{\mathrm{Ex}}$; the r.f. field will be able to momentarily change the temperature $T_{\mathrm{z}}$ of the Zeeman reservoir $\left(\left[S_{x}, S_{z}\right] \neq 0\right)$ without changing that, $T_{E x}$, of the exchange reservoir $\left(\left[S_{x}, \mathbf{s}_{i} \mathbf{s}_{j}\right]=0\right)$. The corresponding density operator is of the form :

$$
\rho=\exp \left(\frac{-\mathscr{H}_{\mathrm{Z}}}{k T_{\mathrm{z}}}-\frac{\mathscr{H}_{\mathrm{Ex}}}{k T_{\mathrm{Ex}}}\right) .
$$

We now introduce the dipolar Hamiltonian $\mathscr{H}_{\mathrm{d}}$; its secular part $\mathscr{H}_{\mathrm{ds}}$ is usually written

$$
J_{\mathrm{ds}}=g^{2} \beta^{2} \sum_{\substack{i<j \\ j}} \frac{A_{i j}+B_{i j}}{r_{i j}^{3}}
$$

where

$$
A_{i j}=s_{z_{i}} s_{z j}\left(1-3 \cos ^{2} \theta_{i j}\right)
$$

and

$$
B_{i j}=\frac{1}{2}\left(1-3 \cos ^{2} \theta_{i j}\right)\left(s_{z_{i}} s_{z_{j}}-\mathbf{s}_{i} \mathbf{s}_{j}\right)
$$

( $\left.{ }^{2}\right)$ Since then, this model has been widely used in the study of the N.M.R. in solid ${ }^{3} \mathrm{He}$ (see [27] and references therein).
Although $\mathscr{H}_{\mathrm{ds}}$ commutes with $\mathscr{H}_{\mathrm{z}}$, it does not commute with $\mathcal{H}_{\mathrm{Ex}}$ (except in the case of spins $\left.1 / 2\right)\left({ }^{3}\right)$ and this has two consequences :

1) it allows energy gained by the Zeeman reservoir from the r.f. field to flow to the exchange reservoir.

2) It allows the spins to relax through the modulation of the $J$ exchange integral by the phonons.

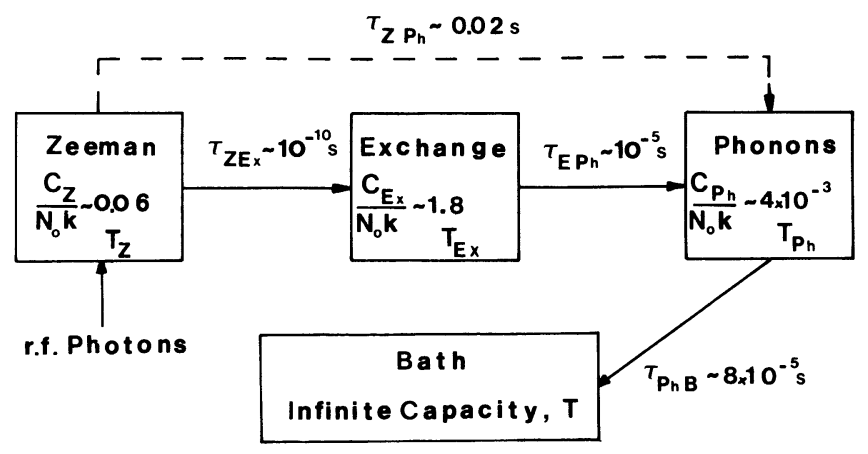

Fig. 13. - Numerical values of the characteristic parameters in the exchange reservoir model, for $\mathrm{Eu}_{0.4} \mathrm{Sr}_{0.6} \mathrm{~S}$, at $4.2 \mathrm{~K}$. Heat capacities : calculated values $\left(N_{0}:\right.$ number of magnetic moments) ; time constants : $\tau_{\mathbf{z E x}}$ was obtained from the linewidth, $\tau_{\mathrm{EPh}}$ was calculated; $\tau_{\mathrm{PhB}}$ comes from our experimental results, taking the existence of a phonon bottleneck into account; the value $\tau_{\mathbf{Z P h}}$ was estimated from Huang's measurements for $\mathrm{Eu}^{2+}$ in $\mathrm{CaF}_{2}$, and shows that the direct Zeeman phonon coupling can be neglected.

We stress that the present situation is opposite to that studied by Provotorov [22], and met for instance in N.M.R. : in that case the dipolar energy is important, and the exchange energy is zero; two reservoirs are then introduced : the Zeeman reservoir, and the dipolar reservoir, associated with $\mathcal{H}_{\mathrm{ds}}$; the flow of energy between these reservoirs is allowed by the nonsecular part of $\mathcal{H}_{\mathrm{d}}$.

It should by now be clear that, when the dipolar and exchange couplings are both important and of the same magnitude, when $s \neq 1 / 2$, it is impossible to introduce three reservoirs (Zeeman, dipolar, exchange) since $\mathscr{H}_{\mathrm{z}}, \mathscr{H}_{\mathrm{ds}}$ and $\mathscr{H}_{\mathrm{Ex}}$ do not commute each with all the others $\left({ }^{4}\right)$.

The assumption $\mathscr{H}_{\mathrm{ds}}<\mathscr{H}_{\mathrm{Ex}}$, essential for the description with Zeeman and exchange reservoirs (hereafter called the Bloembergen case), is also roughly that for exchange narrowing. The Bloembergen model is pictured in figure 13 [20]. As already stated, the energy directly flows from the r.f. field to the Zeeman

$\left({ }^{3}\right)$ One has just to consider whether the commutator $\left[s_{1}^{+} s_{2}^{-}+s_{1}^{-} s_{2}^{+}, s_{z_{1}} s_{z_{2}}\right]-$ hereafter written [ ] - is zero or non zero. It is zero for spins $1 / 2$.

On the contrary, if one considers for instance two spins 1 , it is easy to verify that $\langle 1,-1|[]| 0,0\rangle \neq 0$, which indicates that the commutator is non zero.

(4) Cox et al. [23] have introduced these three reservoirs for spins $1 / 2$. 
reservoir only; as a consequence if $\mathscr{H}_{\mathrm{ds}}$ were zero, the line would be infinitely narrow $\left({ }^{5}\right)$; the presence of a non zero $\mathcal{H}_{\mathrm{ds}}$ allows the flow of energy from the Zeeman to the exchange reservoirs, and leads to a finite linewidth; the time $\tau_{\mathrm{ZEx}}$ characterizing this energy transfer from the Zeeman reservoir must be identified with the time $T_{1}$ associated with the relaxation of $M_{z}$ in B.W.R. theory (Sect. 4.1); in the case of extreme narrowing $T_{1}=T_{2}$, and $\tau_{\mathrm{ZEx}}$ can therefore be directly deduced from the linewidth. Thus, for $\mathrm{Eu}_{0.4} \mathrm{Sr}_{0.6} \mathrm{~S}, \tau_{\mathrm{ZEx}} \sim 10^{-10} \mathrm{~s}$ (Sect. 4.2); this last result is strictly valid only in the paramagnetic region, but can reasonably be taken as an order of magnitude for $\tau_{\mathrm{ZEx}}$ at $4.2 \mathrm{~K}$. As a consequence, even with the shortest r.f. pulses delivered by our magnetron $\left(\tau_{\text {sat }} \sim 1 \mu \mathrm{s}\right)$, the Zeeman and exchange reservoirs will be at the same temperature at the end of the saturating pulse $\left(T_{\mathrm{z}}=T_{\mathrm{Ex}}\right)$.

4.2.2 Calculation of the Zeeman, exchange and phonon heat capacities. - In order to progress in the discussion, we must now make an estimate of the values of the heat capacities associated with the different reservoirs (Fig. 13) $-C_{\mathrm{ph}}$ (phonons), $C_{\mathrm{Z}}$ (Zeeman), $C_{\mathrm{Ex}}$ (exchange) - and of the relaxation times $\tau_{\mathrm{EPh}}$ and $\tau_{\mathrm{PhB}}$ for the exchange-phonon and phononbath couplings.

For an easy comparison, we will calculate the ratio of these heat capacities over $N_{0} k\left(N_{0}\right.$ : number of spins, $k$ : Boltzmann constant), which is dimensionless.

We first consider $C_{\mathrm{Ph}}$, and describe the acoustic phonons with the Debye model; the density for the phonon modes is :

$$
\rho(\omega)=\left\{\begin{array}{cll}
\frac{3 \omega^{2} V}{2 \pi^{2} v^{3}}=\frac{9 N \omega^{2}}{\omega_{D}^{3}} & \text { if } & \omega<\omega_{D} \\
0 & \omega>\omega_{D}
\end{array}\right.
$$

where $N$ is the number of primitive cells in volume $V$, and is also the number of $\mathrm{Eu}^{2+}$ sites, $\omega_{\mathrm{D}}=k T_{\mathrm{D}} / \hbar$ is the Debye angular frequency, and $v=\omega_{\mathrm{D}}\left(\frac{1}{6 \pi^{2}} \frac{V}{N}\right)^{1 / 3}$. The heat capacity for a phonon mode with angular frequency $\omega$ is $C_{\omega}=k \frac{x^{2} \mathrm{e}^{x}}{\left(\mathrm{e}^{x}-1\right)^{2}}$, with $x=\frac{\hbar \omega}{k T}$; if the relaxation of the exchange reservoir to the phonon reservoir takes place via a direct process, and without a transfer of energy from the phonon modes

$\left({ }^{5}\right)$ On the contrary, in the Provotorov case [22] the r.f. energy directly flows to both the Zeeman and dipolar reservoirs $\left(\mathscr{H}_{\text {r.f. }}\right.$ commutes neither with $\mathscr{H}_{\mathrm{z}}$ nor with $\mathscr{H}_{\mathrm{ds}}$, since $\left.\left[S_{x}, S_{z}\right] \neq 0\right)$ : when a photon with energy $\hbar \omega$ is absorbed by a spin, the Zeeman reservoir gets $\hbar \omega_{0}$, and the dipolar reservoir $\hbar\left(\omega-\omega_{0}\right)$; a finite linewidth results, associated with $\mathcal{H}_{\mathrm{ds}}$. in the frequency range $0, \frac{k T_{\mathrm{g}}}{\hbar}$, to the other phonon modes, then the heat capacity is $C_{\mathrm{Ph}}^{\prime} \sim \int_{0}^{k T_{\mathbf{g}} / \hbar} C_{\omega} \rho(\omega) \mathrm{d} \omega$ and is very small; if $T_{\mathrm{g}} \ll T, \frac{C_{\mathrm{Ph}}^{\prime}}{N_{0} k} \simeq 3\left(\frac{N}{N_{0}}\right)\left(\frac{T_{\mathrm{g}}}{T_{\mathrm{D}}}\right)^{3}$, which is temperature independent. Using $\rho=5800 \mathrm{~kg} /$ $\mathrm{m}^{3}, T_{\mathrm{D}}=230 \mathrm{~K}, v=2900 \mathrm{~m} / \mathrm{s}, T_{\mathrm{g}}=1.55 \mathrm{~K}$ for $\mathrm{Eu}_{0.4} \mathrm{Sr}_{0.6} \mathrm{~S}$, then $C_{\mathrm{Ph}}^{\prime} / N_{0} k \sim 2 \times 10^{-6}$. If in the direct process the energy is transferred from the resonant to the non-resonant phonon modes, or if the exchange-phonon relaxation takes place via a Raman process, the phonon heat capacity is $C_{\mathrm{Ph}}=$ $\int_{0}^{\omega_{\mathrm{D}}} C_{\omega} \rho(\omega) \mathrm{d} \omega$; it is well known that if $T \ll T_{\mathrm{D}}$, then $C_{\mathrm{Ph}}=234 N k\left(\frac{T}{T_{\mathrm{D}}}\right)^{3}$; thus for $\mathrm{Eu}_{0.4} \mathrm{Sr}_{0.6} \mathrm{~S}$ and $T \ll T_{\mathrm{D}}, \quad C_{\mathrm{Ph}} / N_{0} k=585\left(\frac{T}{T_{\mathrm{D}}}\right)^{3}$ and at $4.2 \mathrm{~K}$ $C_{\mathrm{Ph}} / N_{0} k \simeq 4 \times 10^{-3}$. We will later see that, in fact, up to $40 \mathrm{~K}$ the direct process is the more efficient, but as explained in 4.2 .3 , the energy is probably given up to the non-resonant modes and from now on we will use this last expression for $C_{\mathrm{Ph}}$.

The heat capacity $C_{\mathrm{Z}}$ of the Zeeman reservoir is the capacity associated with the Zeeman Hamiltonian $\mathscr{H}_{\mathrm{z}}=\sum_{i=1}^{N_{0}} g \beta_{\mathrm{e}} H s_{z_{i}}$ :

$$
C_{\mathrm{Z}} / N_{0} k=x^{2}\left[\frac{1}{4 \operatorname{sh}^{2} \frac{x}{2}}-\left(\frac{2 s+1}{2}\right)^{2} \frac{1}{\operatorname{sh}^{2}\left(\frac{2 s+1}{2}\right) x}\right]
$$

with

$$
x=\frac{g \beta_{\mathrm{e}} H}{k T} ; \text { for } s=7 / 2
$$

$C_{\mathrm{Z}}$ is numerically found to be maximum for $x=1$; thus at $\mathrm{X}$ band and for $T \geqslant 4.2 \mathrm{~K}, x \ll 1$ and the hightemperature approximation is valid :

$$
C_{\mathrm{Z}} / N_{0} k \simeq \frac{s(s+1)}{3} x^{2}
$$

at $4.2 \mathrm{~K}, x=0.10$ and $C_{\mathrm{z}} / N_{0} k \simeq 6 \times 10^{-2}$ for $s=\frac{7}{2}$.

The heat capacity $C_{\mathrm{Ex}_{\mathrm{x}}}$ of the exchange reservoir is associated with the Hamiltonian $\mathscr{H}_{\mathrm{Ex}}=-\sum_{\substack{i \\ j \neq i}} J_{i j} \mathbf{s}_{i} \mathbf{s}_{j}$.

We will make an order of magnitude calculation, with 
the Opechovski high-temperature method [24] : the partition function is

$$
\begin{aligned}
Z & =\operatorname{Tr} \mathrm{e}^{-\beta \mathcal{H}_{\mathrm{Ex}}}= \\
& =(2 s+1)^{N_{0}}\left[1-\beta\left\langle\mathcal{H}_{\mathrm{E}_{\mathbf{x}}}\right\rangle+\frac{\beta^{2}}{2}\left\langle\mathcal{H}_{\mathrm{Ex}_{\mathbf{x}}}^{2}\right\rangle+\cdots\right]
\end{aligned}
$$

where $\beta=\frac{1}{k T}$ and $\left\langle\mathcal{H}_{\mathrm{Ex}}^{n}\right\rangle=(2 s+1)^{-N_{0}} \operatorname{Tr} \mathcal{H}_{\mathrm{Ex}}^{n}$;

then

$$
\log Z=N_{0} \log (2 s+1)+\frac{\beta^{2}}{2}\left\langle\mathcal{H}_{\mathrm{E}_{\mathbf{x}}}^{2}\right\rangle+\cdots
$$

$\left(\left\langle\mathscr{H}_{\mathbf{E x}}\right\rangle\right.$ is zero),

and finally $C_{\mathrm{Ex}} / N_{0} k \simeq \frac{\left\langle\mathcal{H}_{\mathrm{Ex}}^{2}\right\rangle}{(k T)^{2}} ;$ for simplicity we keep the nearest neighbour couplings only; then $C_{\mathrm{Ex}} / N_{0} k \simeq \frac{2}{3} \frac{z s^{2}(s+1)^{2} J^{2}}{(k T)^{2}}$ where $z$ is the number of nearest neighbour magnetic sites, which are considered all occupied; for $\mathrm{Eu}_{0.4} \mathrm{Sr}_{0.6} \mathrm{~S}(z=12$, $S=7 / 2, \quad J / k=0.2 \mathrm{~K}$ ) we will use the previous result, replacing $z$ by $c z \quad(c=0.4)$; then $\frac{C_{\mathrm{Ex}}}{N_{0} k} \simeq \frac{32}{T^{2}}(T: \mathrm{K}) ;$ for $T=4.2 \mathrm{~K}, \frac{C_{\mathrm{Ex}}}{N_{0} k} \simeq 1.8$; thus, when the high temperature approximation is valid, both $C_{\mathrm{z}}$ and $C_{\mathrm{Ex}}$ have a $\frac{1}{T^{2}}$ dependence, and $\frac{C_{\mathrm{Ex}}}{C_{\mathrm{Z}}} \simeq 36$ : the heat capacity of the exchange reservoir in $\mathrm{Eu}_{0.4} \mathrm{Sr}_{0.6} \mathrm{~S}$ is much greater than that of the Zeeman reservoir. At $4.2 \mathrm{~K}$ the heat capacities of the exchange and Zeeman reservoirs overwhelm that of the phonon reservoir, even under the assumption of an exchangephonon Raman process. The exchange and phonon reservoirs have the same heat capacities at $T=16 \mathrm{~K}$.

4.2.3 Calculation of the exchange-phonon relaxation time. - We now discuss the time $\tau_{\mathrm{EPh}}$ describing the rate of transfer from the exchange reservoir to the phonons. We consider that the coupling between these reservoirs originates from the modulation of the exchange coupling by the phonons ( $J$ strongly depends upon the distance $r_{i j}$ between neighbouring spins). We use Griffiths' method [25] for determining $\tau_{\mathrm{EPh}}$, assuming at this stage that the phonon modes act as a thermostat for the spins. The exchange coupling $J_{i j}$ between n.n. spins $s_{i}$ and $s_{j}$ is expanded around the equilibrium position :

$$
\begin{aligned}
& \mathscr{H}=\mathscr{H}_{0}+\mathscr{H}_{1}+\mathscr{H}_{2} \text { where : } \\
& \mathscr{H}_{0}=-2 J \sum_{\langle i j\rangle} \mathbf{s}_{i} \mathbf{s}_{j} \\
& \mathscr{H}_{1}=-\sum_{\langle i j\rangle} \mathbf{s}_{i} \mathbf{s}_{j} O_{i j}=-\sum_{\langle i j\rangle} \mathbf{s}_{i} \mathbf{s}_{j} \sum_{\alpha} 2\left(\frac{\partial J}{\partial x_{i j}^{\alpha}}\right)_{0} u_{i j}^{\alpha}
\end{aligned}
$$

$$
\begin{aligned}
\mathscr{H}_{2}=-\sum_{\langle i j\rangle} \mathbf{s}_{i} \mathbf{s}_{j} P_{i j}= \\
=-\sum_{\langle i j\rangle} \mathbf{s}_{i} \mathbf{s}_{j} \sum_{\alpha, \beta}\left(\frac{\partial^{2} J}{\partial x_{i j}^{\alpha} \partial x_{i j}^{\beta}}\right)_{0} u_{i j}^{\alpha} u_{i j}^{\beta}
\end{aligned}
$$

where the derivatives are taken at the equilibrium positions, and $u_{i j}^{\alpha}$ is the coordinate $\alpha$ for the relative displacements of atoms $i$ and $j$; the summations are over all n.n. pairs. We will assume that $J_{i j} \propto \mathrm{e}^{-\lambda\left|r_{i}-r_{j}\right|}$ $\mathbf{r}_{i}$ and $\mathbf{r}_{j}$ represent the positions of $i$ and $j$ ions.

We first consider the direct process : we use the usual perturbation theory and the interaction representation, introducing the time-dependent operators $A(t)=\mathrm{e}^{i \mathcal{H}_{0 t} / \hbar} A \mathrm{e}^{-i \mathcal{H}_{\mathrm{o} t} / \hbar} . \mathcal{H}_{1}$ is considered a random operator; the probability per unit time for the $\left|M_{S}, n\right\rangle \rightarrow\left|M_{S-1}, n+1\right\rangle$ transition, where the number of phonons in a given mode with angular frequency $\omega$ increases by one unit, is

$\lim _{t \rightarrow \infty} \frac{1}{\hbar^{2} t}\left|\int_{0}^{t}\left\langle M_{S}, n\left|\mathcal{H}_{1}(t)\right| M_{S-1}, n+1\right\rangle \mathrm{e}^{i \omega t} \mathrm{~d} t\right|^{2}$

Neglecting the correlation between spin pairs, one is led to the summation of $\frac{N_{0} z}{2}$ identical terms. Assuming that $\left|T_{\text {Ex }}-T_{0}\right| \ll T_{\text {Ex }}, T_{0}$, and using energy conservation, we find that

$$
\frac{\mathrm{d} T_{\mathrm{Ex}}}{\mathrm{d} t}=\frac{T_{\mathrm{Ph}}-T_{\mathrm{Ex}}}{\tau_{\mathbf{E P h}}}
$$

where

$$
\begin{aligned}
\frac{1}{\tau_{\mathrm{EPh}}} & =\frac{N_{0} z}{2} \sum_{\omega} \frac{\left\langle W_{i j}(\omega)\right\rangle(\hbar \omega)^{2}}{a k} \\
\left(C_{\mathrm{Ex}}\right. & \left.=\frac{a}{T^{2}}\right) .
\end{aligned}
$$

In (8), $\left\langle W_{i j}(\omega)\right\rangle=\frac{1}{\hbar^{2}} \overline{\left.\left\langle n\left|O_{i j}\right| n+1\right\rangle\right|^{2}} F(\omega)$; the bar means the thermal average and

$$
F(\omega)=\int_{-\infty}^{+\infty} f(t) \mathrm{e}^{i \omega t} \mathrm{~d} t
$$

with

$$
f(t)=\operatorname{Tr} \rho_{0} \mathbf{s}_{i}(t) \mathbf{s}_{j}(t) \cdot \mathbf{s}_{i}(0) \mathbf{s}_{j}(0)
$$

$\left(\rho_{0}\right.$ : density operator for $\left.\mathscr{H}_{0}\right)$.

It is possible to know $F(\omega)$ from the calculation of a trace because a time average has been replaced by an ensemble average (ergodic assumption).

In (8) the summation is made over the acoustical phonon modes, using the Debye model presented earlier. In the high-temperature approximation, the 
mean number of phonons in a mode with angular frequency $\omega$ is $\bar{n} \simeq \frac{k T}{\hbar \omega}$ and in this approximation

$$
\left(\frac{1}{\tau_{\mathrm{EPh}}}\right)_{\text {Direct }}=\frac{3 N_{0} z J^{2} \lambda^{2} d^{2}}{4 \pi^{2} \rho v^{5} a} T \int_{0}^{\infty} F(\omega) \omega^{4} \mathrm{~d} \omega .
$$

As it is reasonable to consider that the spectral density $F(\omega)$ has non-zero values in the range 0 , $\omega_{\mathrm{c}}=\frac{k T_{\mathrm{g}}}{\hbar}$, use of this approximation for the phonon modes occupancy is valid for $T \gg T_{\mathrm{g}}$.

The calculation of the fourth moment of $F(\omega)$ has been done with the high-temperature assumption (Appendix 2) and leads to :

$$
\begin{aligned}
& \left(\frac{1}{\tau_{\mathrm{EPh}}}\right)_{\text {Direct }}= \\
& \quad=\frac{8 k T(d \lambda)^{2} J^{4}}{3 \pi \rho v^{5} \hbar^{4}} s(s+1)[16 s(s+1)-3][c(z-1)]^{2}
\end{aligned}
$$

where $d$ is the distance between n.n. $\mathrm{Eu}^{2+}$ sites, and $c$ the $\mathrm{Eu}^{2+}$ concentration. Taking $\rho=5.8 \times$ $10^{3} \mathrm{~kg} / \mathrm{m}^{3}, v=2.9 \times 10^{3} \mathrm{~m} / \mathrm{s}, \quad d=4.2 \times 10^{-10} \mathrm{~m}$, $\lambda=2 \times 10^{10} \mathrm{~m}^{-1}[25], s=\frac{7}{2}, J / k=0.2 \mathrm{~K}$, then $\left(\frac{1}{\tau_{\mathrm{EPh}}}\right)_{\text {Direct }}=2.5 \times 10^{4} \mathrm{~T}\left(\tau_{\mathrm{EPh}} \mathrm{s}, T \mathrm{~K}\right)$.

We now turn to the Raman process, where the spin transition takes place through the emission of a phonon with energy $\hbar \omega$, and the absorption of a phonon with energy $\hbar \omega^{\prime}$; using the same method as for the direct process, one finds an exponential behaviour when $\frac{\hbar\left(\omega-\omega^{\prime}\right)}{k}\left(\frac{1}{T_{\mathrm{Ph}}}-\frac{1}{T_{\mathrm{Ex}}}\right) \ll 1$; if $T_{\mathrm{Ex}}-T_{\mathrm{Ph}} \ll T_{\mathrm{Ex}}, T_{\mathrm{Ph}}$, this condition is equivalent to $T \gg T_{\mathrm{g}}$. One gets then equation (7), where

$\frac{1}{\tau_{\mathrm{EPh}}}=\frac{N_{0} z}{2} \sum_{\omega, \omega^{\prime}} \frac{\left\langle W_{i j}\left(\omega, \omega^{\prime}\right)\right\rangle}{a k} \hbar^{2}\left(\omega-\omega^{\prime}\right)^{2}$.

$$
\text { In (13), }
$$

$$
\begin{aligned}
& \left\langle W_{i j}\left(\omega, \omega^{\prime}\right)\right\rangle= \\
& =\frac{1}{\hbar^{2}} \overline{\left.\left\langle n, n^{\prime}\left|P_{i j}\right| n+1, n^{\prime}-1\right\rangle\right|^{2}} F\left(\omega-\omega^{\prime}\right)\left(^{6}\right) .
\end{aligned}
$$

$\left(^{6}\right)$ The Raman contribution from $\mathscr{H}_{1}$ treated to the second order of perturbation is negligible against that from $\mathrm{He}_{2}$ treated to the first order, for the reason given by Griffiths [25].
One now obtains :

$$
\begin{aligned}
\left(\frac{1}{\tau_{\mathrm{EPh}}}\right)_{\text {Raman }}= & A \int_{0}^{\omega_{\mathrm{D}}} \mathrm{d} \omega^{\prime} g\left(\omega^{\prime}\right) \times \\
& \times \int_{\omega^{\prime}}^{\omega_{\mathrm{D}}}\left(\omega-\omega^{\prime}\right)^{2} F\left(\omega-\omega^{\prime}\right) \mathrm{d} \omega
\end{aligned}
$$

with

$$
A=\frac{9}{128} \frac{N_{0} z\left(J \lambda^{2} d^{2}\right)^{2} \hbar^{2}}{\pi^{4} \rho^{2} v^{10} a k}
$$

and

$$
g\left(\omega^{\prime}\right)=\frac{\omega^{\prime 6} \mathrm{e}^{\hbar \omega^{\prime} / k T}}{\left(\mathrm{e}^{\hbar \omega^{\prime} / k T}-1\right)^{2}} .
$$

Considering that $F(\Omega)$ is non-zero for $0<\Omega<$ $\omega_{\mathrm{c}} \ll \omega_{\mathrm{D}}$, it is finally found that

$$
\left(\frac{1}{\tau_{\mathrm{EPh}}}\right)_{\text {Raman }} \simeq A \int_{0}^{\omega_{\mathrm{D}}} \mathrm{d} \omega^{\prime} g\left(\omega^{\prime}\right) \cdot \int_{0}^{\omega_{c}} \Omega^{2} F(\Omega) \mathrm{d} \Omega .
$$

The first integral is well known in relaxation problems, and will be written $\left(\frac{k T}{\hbar}\right)^{7} I_{6}\left(\frac{T_{\mathrm{D}}}{T}\right)$, the second one is given in appendix 2 . The whole result is :

$$
\begin{aligned}
\left(\frac{1}{\tau_{\mathrm{EPh}}}\right)_{\text {Raman }} \simeq & \frac{3}{16 \pi^{3} \rho^{2} v^{10}}\left(\frac{k T}{\hbar}\right)^{7} I_{6}\left(\frac{T_{\mathrm{D}}}{T}\right) \times \\
& \times\left(J \lambda^{2} d^{2}\right)^{2} s(s+1) \cdot c(z-1) .
\end{aligned}
$$

Using the same numerical values :

$$
\left(\frac{1}{\tau_{\mathrm{EPh}}}\right)_{\text {Raman }} \simeq 7.6 \times 10^{-9} T^{7} I_{6}\left(\frac{T_{\mathrm{D}}}{T}\right)\left(\tau_{\mathrm{EPh}} \mathrm{s}, T \mathrm{~K}\right) .
$$

The values of $\tau_{\mathrm{EPh}}$ calculated for the direct and Raman processes at different temperatures are given in the following table :

\begin{tabular}{rrc}
$T(\mathrm{~K})$ & Direct (s) & Raman (s) \\
\hline 4 & $1 \times 10^{-5}$ & 11 \\
16 & $2.5 \times 10^{-6}$ & $7 \times 10^{-4}$ \\
30 & $1.3 \times 10^{-6}$ & $8 \times 10^{-6}$
\end{tabular}

The two processes have the same efficiency around $40 \mathrm{~K}$. Then $T_{1} \sim 1 \mu \mathrm{s}$.

4.2.4 Relaxation of the spin-phonon system. - It is now possible to give a simplified picture of our system, valid for $t \gg 10^{-10} \mathrm{~s}$, a condition always met in our dynamical experiments :

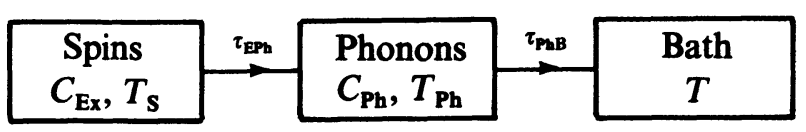


Our experimental result $\left(\tau_{\text {obs }} \sim 40 \mathrm{~ms}\right.$ at $\left.4.2 \mathrm{~K}\right)$ and the fact that at this temperature $\frac{C_{\mathrm{Ex}}}{C_{\mathrm{ph}}} \sim 500$ suggest the existence of a phonon bottleneck. It is well known [19] that the evolution of the spin temperature $T_{\mathrm{s}}$ towards $T$, the temperature of the bath, is then not exponential, but proceeds through two steps : a fast thermalization of the phonons with the spins system (time constant $\tau^{\prime} \simeq \frac{C_{\mathrm{Ph}}}{C_{\mathrm{Ex}}} \tau_{\mathrm{EPh}} \simeq 20 \mathrm{~ns}$ at $4.2 \mathrm{~K}$ ), followed by a far slower evolution of the common temperature towards $T$ (time constant $\left.\tau^{\prime \prime} \simeq \frac{C_{\mathrm{Ex}}}{C_{\mathrm{Ph}}} \tau_{\mathrm{PhB}}(17)\right)\left({ }^{7}\right)$. If the lifetime $\tau_{\mathbf{P h}}$ of the phonons were limited by the inelastic scattering at the crystal boundaries, then $\tau_{\mathrm{PhB}} \simeq \tau_{\mathrm{Ph}} \sim \frac{L}{2 v} \sim 1.4 \times 10^{-7} \mathrm{~s}$. The longer time constant should then be $\tau^{\prime \prime} \sim 7 \times 10^{-5} \mathrm{~s}$ instead of $4 \times 10^{-2} \mathrm{~s}$ as found in our experiments. One could then imagine that because of the mismatch at the boundaries, a phonon makes $Q$ reflexions before being absorbed, which in our case leads to $Q \sim 500$. In fact, it seems more realistic to think that a given phonon has a much greater chance to be diffused by scattering centres (spins, as their concentration is high, or others defects) than to reach the crystal boundary; this leads to a poorer transfer of energy to the bath, and to a lengthening of the time necessary for the spin-phonon system to reach its equilibrium with the helium bath : if this scattering process takes place, then from equation (17) and our experimental result, $\tau_{\mathrm{PhB}} \sim 8 \times 10^{-5} \mathrm{~s}$. One can crudely describe the diffusion of energy from the spins in the centre of the sample to the bath as a one-dimensional random walk problem, supposing that the scattering centres are regularily spaced; the energy is then given up in a time $\tau_{\mathrm{PhB}}=N \tau_{\mathrm{c}}$ where $\tau_{\mathrm{c}}$ is the mean time between two collisions, and $N$ the number of collisions. Moreover $\tau_{\mathrm{PhB}}=\sqrt{N} \frac{L}{2 v}$ which leads to $\sqrt{N}=570$ and $\tau_{\mathrm{c}}=0.25 \times 10^{-9} \mathrm{~s}$.

Our present interpretation seems corroborated by thermal conductivity measurements [14], which have been explained by assuming the existence of scattering centres; the lifetime of the phonons with energy $\hbar \omega \sim 3 k T$ deduced from these measurements should then be $\tau_{c} \sim 5 \times 10^{-9} \mathrm{~s}$ at $4.2 \mathrm{~K}$. Thus both interpretations are in qualitative agreement, and the difference in the $\tau_{c}$ value is not surprising remembering the crudeness of our model.

( 7 ) At $4.2 \mathrm{~K}$, if the exchange-phonon relaxation took place without the transfer of energy from the resonant to the non-resonant phonons, the $\tau^{\prime \prime}$ value would be $\tau^{\prime \prime} \simeq \frac{C_{\mathrm{Ex}}}{C_{\mathrm{Ph}}^{\prime}} \tau_{\mathrm{EPh}}$ with $C_{\mathrm{Ex}} / C_{\mathrm{Ph}}^{\prime} \simeq 10^{6}$ which would lead to $\tau^{\prime \prime} \sim 10 \mathrm{~s}$; in fact, in such a long time, the energy must diffuse throughout the whole phonon spectrum. This justifies the use of $C_{\mathrm{Ph}}$ instead of $C_{\mathrm{Ph}}^{\prime}$ in our discussion.
4.3 THE SPIN TEMPERATURE RAISE : PREDICTIONS AND OBSERVATIONS. - Before concluding, it is interesting to use the model for calculating an order of magnitude of the spin temperature increase at $4.2 \mathrm{~K}$, and compare it with the experimental results. The temperatures obey the following system of equations $[20,26]$ :

$$
\begin{gathered}
C_{\mathrm{Z}} \dot{T}_{\mathrm{z}}=\frac{\alpha}{T_{\mathrm{Z}}}+\frac{C_{\mathrm{Z}}\left(T_{\mathrm{Ex}}-T_{\mathrm{Z}}\right)}{\tau_{\mathrm{ZEx}}} \\
C_{\mathrm{Ex}} \dot{T}_{\mathrm{Ex}}=-\frac{C_{\mathrm{Z}}\left(T_{\mathrm{Ex}}-T_{\mathrm{Z}}\right)}{\tau_{\mathrm{ZEx}}}+ \\
\quad+\frac{C_{\mathrm{Ex}}\left(T_{\mathrm{Ph}}-T_{\mathrm{Ex}}\right)}{\tau_{\mathrm{EPh}}} \\
C_{\mathrm{Ph}} \dot{T}_{\mathrm{Ph}}=-\frac{C_{\mathrm{Ex}}\left(T_{\mathrm{Ph}}-T_{\mathrm{Ex}}\right)}{\tau_{\mathrm{EPh}}}+ \\
+\frac{C_{\mathrm{Ph}}\left(T_{\mathrm{B}}-T_{\mathrm{Ph}}\right)}{\tau_{\mathrm{PhB}}}
\end{gathered}
$$

where $\alpha$ is defined as following : the energy $\mathrm{d} W$ absorbed by the Zeeman reservoir from the photons during $\mathrm{d} t$ is :

$$
\mathrm{d} W=\hbar \omega \sum_{m_{\mathrm{s}}=-s}^{s-1} U_{m_{\mathrm{s}} \rightarrow m_{\mathrm{s}}+1}\left(n_{m_{\mathrm{s}}}-n_{m_{\mathrm{s}}+1}\right) N_{0} \mathrm{~d} t
$$

( $n_{m_{\mathrm{s}}}$ is the population of level $m_{\mathrm{s}}, U_{m_{\mathrm{s}} \rightarrow m_{\mathrm{s}}+1}$ the transition probability for the r.f. field); in the high-temperature approximation, assuming a Lorentzian line shape (width at half-height $\left.\Delta \omega, T_{2}=\frac{2}{\Delta \omega}\right)$, it is easily found that

$$
\begin{aligned}
\frac{\mathrm{d} W}{\mathrm{~d} t}=\left(\gamma H_{1}\right)^{2} \frac{(\hbar \omega)^{2}}{k T_{\mathrm{z}}} & T_{2} \frac{s(s+1)}{3} N_{0}= \\
& =\frac{\alpha}{T_{\mathrm{z}}}=\left(\gamma H_{1}\right)^{2} T_{2} C_{\mathrm{Z}} T_{\mathrm{z}}
\end{aligned}
$$

- In the stationary regime :

$$
\frac{\alpha}{T_{\mathbf{Z}}}=\frac{T_{\mathbf{Z}}-T_{\mathbf{B}}}{\sum_{i=\mathbf{Z}, \mathbf{E x}, \mathbf{P h}} \tau_{i} / C_{i}}
$$

in our system, the most important term in the sum is that for the phonons so :

$$
\frac{T_{\mathrm{Z}}-T_{\mathrm{B}}}{T_{\mathrm{Z}}} \simeq \frac{\alpha}{T_{\mathrm{Z}}^{2}} \frac{\tau_{\mathrm{PhB}}}{C_{\mathrm{Ph}}}=\left(\gamma H_{1}\right)^{2} \tau_{\mathrm{PhB}} T_{2} \frac{C_{\mathrm{Z}}}{C_{\mathrm{Ph}}}
$$

one can say that in continuous saturation experiments the effective saturation factor is :

$$
s_{\mathrm{eff}}^{2}=\left(\gamma H_{1}\right)^{2} \tau_{\mathrm{Ph}} T_{2} \frac{C_{\mathrm{Z}}}{C_{\mathrm{Ph}}} .
$$

In our experiments, if $P=0.1 \mathrm{~W}, H=0.43 \mathrm{G}$; using 
$T_{2}=10^{-10} \mathrm{~s}, \tau_{\mathrm{Ph}}=8 \times 10^{-5} \mathrm{~s}, C_{\mathrm{Z}} / C_{\mathrm{Ph}}=2 \times$ $10^{4} T^{-5}$, then $T_{\mathrm{Z}}^{4}\left(T_{\mathrm{Z}}-T_{\mathrm{B}}\right) \simeq 9000$, which leads to $\Delta T_{\mathrm{z}} \simeq 2.7 \mathrm{~K}$. This is in fairly good agreement with our experimental finding $\Delta T_{\mathrm{S}} \simeq 0.9 \mathrm{~K}$, considering that $\tau_{\mathrm{PhB}}$ is only approximately known and probably depends upon temperature, and that equations (18) are valid only for small temperatures differences.

- At the end of a saturating pulse with $P_{\text {sat }}=1 \mathrm{~mW}$, $\tau_{\text {sat }}=10 \mathrm{~ms}$, we experimentally found that $\Delta T_{\mathrm{s}} \simeq$ $0.04 \mathrm{~K}$; this was determined by measuring the relative variation of the absorption signal at the end of the pulse $\left(\frac{\Delta \chi^{\prime \prime}}{\chi^{\prime \prime}} \sim 0.03\right)$ and using the results of figure 2 . In order to compare this result with the predictions of the model, we consider that during the r.f. pulse the spin system is isolated from the phonons; strictly speaking, the results of the present treatment are valid only if $\tau_{\text {sat }}<\tau_{\mathrm{EPh}}$; in fact they are valid as long as $\tau_{\text {sat }}<\tau^{\prime \prime}\left(\tau^{\prime \prime} \sim 40 \mathrm{~ms}\right)$, since $C_{\mathrm{Ph}} \ll C_{\mathbf{E x}}$.

When a r.f. pulse is applied, within $10^{-10} \mathrm{~s}$ the exchange and the Zeeman systems thermalize, then the spin temperature $T_{\mathrm{S}}$ of the whole spin system increases with time according to :

$$
\frac{\mathrm{d} W}{\mathrm{~d} t}=\frac{\alpha}{T_{\mathrm{S}}} \simeq C_{\mathrm{Ex}} \frac{\mathrm{d} T_{\mathrm{S}}}{\mathrm{d} t}=\frac{a}{T_{\mathrm{S}}^{2}} \frac{\mathrm{d} T_{\mathrm{S}}}{\mathrm{d} t}
$$

for a small variation, the relative spin temperature increase $\Delta T_{\mathrm{S}} / T_{\mathrm{S}}$ at the end of a pulse of duration $\tau_{\text {sat }}$ is then

$$
\frac{\Delta T_{\mathrm{S}}}{T_{\mathrm{S}}} \simeq \frac{\alpha}{a} \tau_{\mathrm{sat}}=\left(\gamma H_{1}\right)^{2} T_{2} \tau_{\mathrm{sat}} \frac{C_{\mathrm{Z}}}{C_{\mathrm{Ex}}}
$$

with $P=1 \mathrm{~mW}\left(H_{1}=0.04 \mathrm{G}\right), T_{2}=10^{-10} \mathrm{~s}$, $\tau_{\text {sat }}=10^{-2} \mathrm{~s}, C_{\mathrm{z}} / C_{\mathrm{Ex}}=0.033$, then $\Delta T_{\mathrm{s}} \simeq 0.08 \mathrm{~K}$ which compares favourably with our experimental result.

\section{Conclusion.}

Most experimental results presented in this paper were obtained at $4.2 \mathrm{~K}$, and some around $1.6 \mathrm{~K}$. We limited the discussion to the $4.2 \mathrm{~K}$ results. We were able to use the exchange reservoir model, valid when $T \gtrsim T_{\mathrm{g}}$ (in $\mathrm{Eu}_{0.4} \mathrm{Sr}_{0.6} \mathrm{~S}, T_{\mathrm{g}}=2.3 \mathrm{~K}$ at $10 \mathrm{GHz}$ ). We summarize the most salient features which have emerged from our experimental and theoretical work (Fig. 13) together with some open questions.

When the spins are submitted to a perturbating r.f. pulse, the energy is absorbed by the Zeeman reservoir. This energy is then rapidly (within $10^{-10} \mathrm{~s}$ ) transferred to the Exchange reservoir, via the dipolar interactions. In practical situations, the Zeeman and Exchange reservoirs are thus at the same temperature at the end of the r.f. pulse; the heat capacity of this composite spin system is nearly that of the exchange reservoir.

The energy transfer from the spins to the phonons takes place via the modulation of the exchange coupling by the phonons, and not via the modulation of the crystal field by the phonons, a process effective in dilute insulating paramagnets. At $4.2 \mathrm{~K}$, the heat capacity of the spin system is far greater than that of the phonons, which leads to a phonon bottleneck : the phonons rapidly (typically within $2 \times 10^{-8} \mathrm{~s}$ ) reach the spin temperature, then the temperature of the whole spin-phonon system slowly $\left(4 \times 10^{-2} \mathrm{~s}\right)$ reaches that of the helium bath. During this thermalization process, the phonons are scattered by centres whose origin is still unknown (e.g. $\mathrm{Eu}^{2+}$ spins, or lattice defects). The mechanism by which the energy is transferred from the resonant to the non-resonant phonons has still to be found ( $U$-processes are generally ineffective at $4.2 \mathrm{~K}$ ).

Near $T_{\mathrm{g}}$, the value of the resonance field depends upon the spin temperature, and the transient signal observed after a r.f. pulse is finally directly related to the relaxation of this common spin-phonon temperature. We were able to measure the spin temperature increase in both continuous and pulse saturation E.S.R., which agrees with the predictions of the reservoir model. The origin of the dependence of the resonance field versus the spin temperature is still not well understood; in insulating spin-glasses it is usual to focus upon exchange interactions between n. and n.n. neighbours, but this paper shows that the dipolar interactions play an important role and should be considered on the same footing as the exchange coupling

We finally stress that the effects previously described should be found in other insulating spinglasses besides EuSrS, or in semiconducting spinglasses like CdMnTe or ZnMnTe because of their strong exchange capacity compared to that of the phonons, at low temperatures.

\section{Acknowledgments.}

We would like to thank Dr. H. Maletta, who kindly lent us the samples, and Dr. P. Monod, who encouraged this work and made fruitful comments.

\section{Appendix 1.}

In a cylindrical $\mathrm{TE}_{011}$ cavity (length $L$, diameter $D$, volume $V$ ) the r.f. field $2 H_{1} \cos \omega t$ in the middle of the cavity axis is such that :

$$
H_{1}^{2}=\frac{P Q_{0}}{\omega \mu_{0} V J_{0}^{2}\left(\frac{k_{1} D}{2}\right)\left[1+\left(\frac{k_{1}}{k_{3}}\right)^{2}\right]}
$$

where $P$ is the r.f. power dissipated in the cavity, $\omega$ the angular frequency, $\mu_{0}$ the vacuum permeability, $\frac{k_{1} D}{2}=3.832$ is the first zero of the derivative of $J_{0}(x)$, 
the Bessel function of zero order, and $k_{3}=\frac{\pi}{L}$; for $D=46 \mathrm{~mm}$ and $\frac{\omega}{2 \pi}=9.3 \mathrm{GHz}$ this leads to $\left(\mu_{0} H_{1}\right)^{2}$ $=1.87 \times 10^{-12} Q_{0} P\left(P W, \mu_{0} H_{1} \mathrm{~Wb} / \mathrm{m}^{2}\right)$. In our experiments, the cavity is at or near critical coupling, and $P$ is nearly equal to the incident power.

\section{Appendix 2.}

As $F(\omega)$ is the Fourier transform of $f(t)$,

$$
\begin{aligned}
& \int_{0}^{\infty} F(\omega) \omega^{2 p} \mathrm{~d} \omega=\pi(-1)^{p}\left(\frac{\partial^{2 p} f}{\partial t^{2 p}}\right)_{0} \\
& \left(\frac{\partial^{2} f}{\partial t^{2}}\right)_{0}=\frac{1}{\hbar^{2}}\left\langle\left[\mathcal{H}_{0}, \mathbf{s}_{i} \mathbf{s}_{j}\right]^{2}\right\rangle \\
& \left(\frac{\partial^{4} f}{\partial t^{4}}\right)_{0}=\frac{1}{\hbar^{4}}\left\langle\left[\mathcal{H}_{0},\left[\mathcal{H}_{0}, \mathbf{s}_{i} \mathbf{s}_{j}\right]\right]^{2}\right\rangle
\end{aligned}
$$

where \langle\rangle means thermal average. This thermal average appears because each level of the spin system has to be weighted by its Boltzmann factor. When
$T \gg T_{\mathrm{g}}$, these levels are considered to be equally populated. One is then led to the calculation of a trace.

- For a linear chain

$$
\frac{\operatorname{Tr}\left[\mathscr{H}_{0}, \mathbf{s}_{1} \mathbf{s}_{2}\right]^{2}}{\operatorname{Tr} 1}=\frac{16 J^{2} t^{3}}{9} \text { with } t=s(s+1) .
$$

For a cubic lattice and $z$ magnetic neighbours this result must be multiplied by $(z-1)$.

- For a linear chain

$$
\frac{\operatorname{Tr}\left[\mathcal{H}_{0},\left[\mathcal{H}_{0}, \mathbf{s}_{1} \mathbf{s}_{2}\right]\right]^{2}}{\operatorname{Tr} 1}=\frac{64}{27} J^{4} t^{3}(16 t-3) .
$$

The corresponding calculation for a cubic lattice and arbitrary $s$ is much more complicated ; a good approximation is to multiply the previous result by $(z-1)^{2}$ (for $s=\frac{1}{2}$ and a simple cubic lattice, Griffiths [25] has found that the error is less than $4 \%$ ).

The calculations of the various traces are simplified by introducing the quantities $(i \pm j)=\frac{1}{2}\left(s_{i}^{+} s_{j}^{-} \pm\right.$ $\left.s_{i}^{-} s_{j}^{+}\right)$then $\mathbf{s}_{1} \mathbf{s}_{2}=(1-2)+s_{1 z} s_{2 z}$ and $\left[\mathbf{s}_{1} \mathbf{s}_{2}, \mathbf{s}_{2} \mathbf{s}_{3}\right]=$ $(1-2) s_{3 z}+(2 \succ 3) s_{1 z}+(3-1) s_{2 z}$.

\section{References}

[1] Cannella, V., Mydosh, J. A., Phys. Rev. B 6 (1972) 4220.

[2] OWen, J., Browne, M., Knight, W. D., Kittel, C., Phys. Rev. 102 (1956) 1501.

[3] OWen, J., Browne, M. E., ARP, V., KIP, A. F., J. Phys. Chem. Solids 2 (1957) 85.

[4] Mozurkewich, G., Elliott, J. H., Hardiman, M., Orbach, R., Phys. Rev. B 29 (1984) 278.

[5] Deville, A., Arzoumanian, C., Gaillard, B., BlanChard, C., Jamet, J. P., Maletta, H., J. Physique 42 (1981) 1641.

[6] Saslow, W. M., Phys. Rev. B 22 (1980) 1174.

[7] Schultz, S., Gullikson, E. M., Fredkin, D. R., Tovar, M., Phys. Rev. Lett. 45 (1980) 1508.

[8] Becker, K. W., Phys. Rev. B 26 (1982) 2394.

[9] Levy, P. M., Morgan-Pond, C., Raghavan, R., (to be published).

[10a] Chen, M. C., Slichter, C. P., Phys. Rev. B 27 (1983) 278.

[10b] Alloul, H., Bloyet, D., Varoquaux, E., Proceedings of the 14th International Conference on Low Temperature Physics, Otanieui, Finland (1975), edited by M. Kursuis and M. Vuorino (North Holland, Amsterdam) 1975, vol. 3, p. 386.

[11] Bohn, H. G., ZINn, W., Dorner, B., Kollinar, A., Phys. Rev. B 22 (1980) 5447.

[12] $a$ Blanc, C., Deville, A., Hervé, J., Roger G., J. Phys. E 5 (1972) 343. b Deville, A., Blanchard, C., Gaillard, B., Gayda, J. P., J. Physique 36 (1975) 1151.

[13] Huang, C. Y., Phys. Rev. 139 (1965) A 241.

[14] Arzoumanian, C., De Goi:R, A. M., Salce, B., J. Physique Lett. 44 (1983) L-39.

[15] Moruzzi, V. L., Teaney, D. T., Solid State Commun. 1 (1963) 127.

[16] SLICHTER, C. P., Principles of magnetic resonance (Harper and Row, New York) 1964.

[17] Abragam, A., Les principes du magnétisme nucléaire (P.U.F.) 1961.

[18] Lloyd, J. P., Pake, G. E., Phys. Rev. 92 (1954) 1576.

[19] Abragam, A., Bleaney, B., Résonance paramagnétique des ions de transition (P.U.F.) 1971.

[20] Bloembergen, N., Wang, S., Phys. Rev. 93 (1954) 72.

[21] Van Vleck, J. H., Nuovo Cimento, Sup. Ser. X, VI 3 (1957) 1081.

[22] Provotorov, B. N., Soviet Phys. J.E.T.P. 14 (1962) 1126.

[23] Cox, S.F. J., Gill, J. C., Wharmly, D. O., J. Phys. C : Solid State Phys. 4 (1971) 371.

[24] Herpin, A., Théorie du magnétisme (P.U.F.) 1968, p. 309.

[25] Griffiths, R.B., Phys. Rev. 124 (1961) 1023.

[26] Scott, P. L., JefFries, C. D., Phys. Rev. 127 (1962) 32.

[27] Abragam, A., Goldman, M., Nuclear magnetism : order and disorder (Clarendon Press) 1982. 